\title{
HI observations of loose galaxy groups
}

\section{Data and global properties}

\author{
W. van Driel ${ }^{1,2}$, P. Marcum ${ }^{3}$, J. S. Gallagher III $^{4}$, E. Wilcots ${ }^{4}$, C. Guidoux ${ }^{5}$, and D. Monnier Ragaigne ${ }^{1}$ \\ 1 DAEC, UMR CNRS 8631, Observatoire de Paris, Section de Meudon, 5 place Jules Janssen, \\ 92195 Meudon Cedex, France \\ e-mail: wim.vandriel@obspm.fr; delphine.ragaigne@obspm.fr \\ 2 Unité Scientifique Nançay, USR CNRS B704, Observatoire de Paris, 18330 Nançay, France \\ 3 Department of Physics and Astronomy, Texas Christian University, Box 298840, Fort Worth, TX 76129, USA \\ e-mail: p.marcum@tcu.edu \\ 4 Astronomy Department, University of Madison-Wisconsin, 475 N. Charter St., Madison WI 53706-1582, USA \\ e-mail: jsg@astro.wisc.edu; ewilcots@astro.wisc.edu \\ ${ }^{5}$ Faculté des Sciences et des Techniques, Université de Tours, Avenue Monge, 37000 Tours, France
}

Received 24 July 2001 / Accepted 4 September 2001

\begin{abstract}
At Nançay, 21-cm H i line observations were made of 15 spiral-dominated loose groups of galaxies, divided into two samples: an "interacting" sample containing at least one pair of interacting galaxies, and a "control" sample having no optical evidence of interactions or morphological disturbances among the group members. The interacting sample consists of 62 galaxies representing 9 different groups, and the control sample contains 40 galaxies representing 6 groups. Of the 91 galaxy and galaxy pairs observed, 74 were detected, while upper limits were placed on the remaining 17 objects. These homogeneous H I data, which will be used in future analyses, provide comparative information on the H I content of groups and serve as a probe of the vicinity of the target spirals for $\mathrm{H}$ I clouds or very low surface brightness gas-rich galaxies.
\end{abstract}

Key words. galaxies: distances and redshifts - galaxies: general - galaxies: interactions - galaxies: ISM radio lines: galaxies

\section{Introduction}

Sufficient evidence now exists to support the idea that at least some groups of galaxies are gravitationally bound entities, and are not merely chance projections or transient clumpiness (Hernquist et al. 1995; Ostriker et al. 1995) in the background of galaxies (Mamon 1986; Rose 1977, 1979). The majority of this evidence comes from X-ray observations which have revealed that some groups are enveloped in a diffuse, hot intra-group gas (Bahcall et al. 1984; Biermann \& Kronberg 1984; Mahdavi et al. 1997; Fukazawa et al. 1996; Mulchaey et al. 1996; Davis et al. 1999), analogous to the hot medium seen within dense clusters. The higher the elliptical population within a group, the higher the probability that the group will have an intragroup medium that is detectable by ROSAT (Mulchaey et al. 1996; Mahdavi et al. 1997).

Send offprint requests to: W. van Driel, e-mail: wim.vandriel@obspm.fr
However, the non-detection of a hot, intragroup medium within spiral-rich galaxy groups does not preclude those groups from being real physical systems. The dynamical evolution of a bound group will influence the temperature of the intragroup medium (Mulchaey \& Zabludoff 1998), analogous to correlations such as that seen between $L_{x}, T_{x}$ and velocity dispersion in galaxy clusters (Xue \& Wu 2000). Some studies (Marcum 1994; Hickson et al. 1988; Nolthenius 1993) show that, on average, spiral-rich groups have lower velocity dispersions as compared to elliptical-dominated systems. This reduction in the thermal energy being deposited into the intragroup medium of spiral-rich groups predicts that the extant intergalactic gas in these systems would most likely exist mainly in the form of neutral hydrogen. Indeed, some groups have been found to be rich in neutral hydrogen which is tied up in either low surface brightness dwarfs (Gallagher et al. 1995) or H I clouds (e.g., Schneider et al. 1986b; Hoffman et al. 1992). 
Table 1. Group properties.

\begin{tabular}{|c|c|c|c|c|c|c|c|}
\hline $\begin{array}{l}\text { GH } \\
\text { No. } \\
(1)\end{array}$ & $\begin{array}{l}\text { NGal } \\
(2)\end{array}$ & $\begin{array}{l}\text { RA } \\
\quad(2000.0) \\
\quad(3)\end{array}$ & Dec. & $\begin{array}{r}<V_{\text {vir }}> \\
\left(\mathrm{km} \mathrm{s}^{-1}\right) \\
(4)\end{array}$ & $\begin{array}{r}\sigma_{V} \\
\left(\mathrm{kms}^{-1}\right) \\
(5)\end{array}$ & $\begin{array}{r}d \\
(\mathrm{Mpc}) \\
(6)\end{array}$ & $\begin{array}{r}B_{\mathrm{T}} \\
(\mathrm{mag}) \\
(7)\end{array}$ \\
\hline \multicolumn{8}{|c|}{ Interacting group sample } \\
\hline 45 & 4 & 0916.8 & 4117 & 1833 & 150 & 24.4 & 12.4 \\
\hline 58 & 10 & 1019.5 & 2046 & 1319 & 217 & 17.6 & 10.1 \\
\hline 67 & 8 & 1051.4 & 3335 & 1814 & 174 & 24.2 & 10.7 \\
\hline 86 & 3 & 1137.6 & 3209 & 2806 & 41 & 37.4 & 12.4 \\
\hline 92 & 11 & 1154.9 & 2529 & 4361 & 518 & 58.1 & 11.8 \\
\hline 126 & 6 & 1356.1 & 3740 & 3532 & 357 & 47.1 & 11.3 \\
\hline 141 & 13 & 1423.9 & 3601 & 3683 & 461 & 49.1 & 10.6 \\
\hline 153 & 3 & 1526.3 & 4117 & 2790 & 45 & 37.2 & 12.7 \\
\hline 156 & 4 & 1534.8 & 1530 & 2040 & 93 & 27.2 & 11.6 \\
\hline \multicolumn{8}{|c|}{ Control group sample } \\
\hline 49 & 3 & 0950.5 & 4344 & 4889 & 65 & 65.2 & 12.3 \\
\hline 57 & 4 & 1013.6 & 0321 & 1248 & 60 & 16.6 & 10.6 \\
\hline 89 & 7 & 1142.5 & 0946 & 6118 & 180 & 81.6 & 11.8 \\
\hline 118 & 3 & 1325.4 & 3614 & 5537 & 254 & 73.8 & 12.7 \\
\hline 123 & 17 & 1351.3 & 4053 & 2600 & 158 & 34.6 & 9.8 \\
\hline 155 & 6 & 1534.0 & 4316 & 5933 & 149 & 79.1 & 12.1 \\
\hline
\end{tabular}

Note: mean group velocities $V_{\text {vir }}$ were corrected for Virgocentric infall, and $H_{0}=75 \mathrm{~km} \mathrm{~s}^{-1} \mathrm{Mpc}^{-1}$ was assumed.

The dynamical evolution of a loose group is undoubtedly actuated by multiple minor mergers between these gas-rich satellites and larger group members (Haynes et al. 2000), as well as the more dramatic interactions between the large mass galaxies. While the ramifications of such encounters can be found across the spectrum (such as $\mathrm{H} \alpha$, $B$ and thermal infrared luminosity enhancements resulting from the ensuing star formation activity, and optical morphological signatures such as tidal tails and bridges), there is evidence that the disruptions in the gaseous disk is long-lived. For example, the presence of X-shaped structures seen in some peculiar S0 galaxies (Mihos et al. 1995), and the counter-rotating disks seen in some early-type spirals (Corsini et al. 1998; Jore et al. 1996) in otherwise optically normal-looking galaxies is interpreted as the aftermath of minor mergers. The outer regions of gas disks are vulnerable to warps and other distortions created by a close encounter with a passing galaxy, and are not likely to rebound quickly once disturbed. The denser regions of a galaxy cluster environment, where galaxy interactions likely occur with high frequency, impact the Hi properties of the cluster members even more severely: H I disks in galaxies located closest to the cluster core are more likely to be gas-deficient, truncated and asymmetrical. These trends are found even in loose clusters (Chamaraux et al. 1980; Haynes et al. 1985). Thus, peculiarities in the H I properties of a galaxy serve as a "fossil" record of past galaxy-galaxy interactions. Groups of galaxies which are dynamically evolved systems, whose members have experienced multiple disruptive interactions, would be expected to harbor a higher frequency of $\mathrm{HI}$ peculiarities.
Therefore, a comparative analysis of H I properties can be used as relative dynamical "age" indicator for groups of galaxies.

Based on the idea that the neutral hydrogen properties is sensitive to environment, our main motivation for this single-dish H I line observational study of loose groups is to test whether "interacting" groups, which we define as groups hosting at least one pair of optically disturbed interacting galaxies, show evidence for prolonged histories of galaxy-galaxy interactions among the other group members. In the "interacting" groups, most of the galaxy members (with, of course, the exception of the interacting pair itself) show no unusual optical features indicative of past tidal interactions. Either (1) the group is a truly youthful kinematical system, having not yet experienced multiple tidal interactions within the system, or (2) the aftermath of previous galaxy-galaxy interactions among the other group members has left no signatures which are still optically visible. A comparative analysis of asymmetries in the H I line profile shapes (however with caution: see Richter \& Sancisi 1994) and the total H I content for the galaxy groups can help distinguish between these two possibilities. Understanding the kinematical evolution of galaxy groups is particularly important, in light of HST observations of galaxy groups at very high redshift.

Though oft-cited H I mappings of galaxy groups frequently show tidal $\mathrm{HI}$ features, it would be misleading to draw the conclusion from these examples that such features are commonplace. Generally, these observations cover only the inner parts of groups, often centered on an interacting galaxy pair. Examples are the Arecibo maps of 
groups by Haynes et al. (1981), showing tidal features from at least one group member in 6 of the 15 groups mapped, and the VLA maps of the M81 group by Yun et al. (1994). Observations searching for H I throughout the volume covered by groups are rare, due to the large apparent size of nearby groups. Systematic searches for H I clouds in groups were made at Arecibo by Lo \& Sargent (1978) in the nearby M 81, NGC 1023 and CVnI groups (the latter was also covered in the Nançay blind $\mathrm{H}$ I line search by Kraan-Korteweg 1999) and by Zwaan $(2000,2000)$ in 6 groups at redshifts of $1800-3000 \mathrm{~km} \mathrm{~s}^{-1}$ (NGC 5798, $5962,5970,6278,6500$ and 6574) with properties similar to those of the Local Group of galaxies. All H I emission features detected in these searches could unambiguously be associated to optically identified galaxies or to a previously known H I tidal feature in the NGC 6500/01 pair. The $5 \sigma \mathrm{HI}$ mass detection limit for an $\mathrm{H}$ I cloud with a linewidth of $10 \mathrm{~km} \mathrm{~s}^{-1}$ is about $8 \times 10^{6} M_{\odot}$ at the average distance of $30 \mathrm{Mpc}$ (for $H_{0}=75 \mathrm{~km} \mathrm{~s}^{-1} \mathrm{Mpc}^{-1}$ ) for the groups in Zwaans' study. For comparison, the detection limit of the present $\mathrm{H}$ I survey is about 4 times higher.

In this paper we present a new homogeneous set of $\mathrm{HI}$ 21-cm line observations for galaxies in 15 loose groups: 9 with at least one pair of strongly interacting galaxies and 6 without optical indicators of tidal interactions. The galaxy group sample selection is described in Sect. 2, where the basic optical properties of the program galaxies are listed as well. The observations and data reduction are described in Sect. 3, and the H I results are presented in Sect. 4, including notes to invidual galaxies for the interpretation of the 21-cm line data and a comparison of the observed global line parameters with published values. These H I data will, together with our optical and near-infrared data on the groups, provide the basis for a discussion of evolution within galaxy groups that is planned for a future paper (Marcum et al., in preparation).

\section{The observed galaxy group sample}

We used the Nançay telescope for a pencil beam H line study of spiral galaxies and their surroundings in 15 loose groups. Program galaxies in our survey are members of groups selected from the Geller \& Huchra (1983, GH83) group catalogue. Two different samples for comparison have been selected:

1. an "interacting" sample of 9 groups which were purposely chosen to contain at least one pair of strongly interacting Arp-type galaxies, as indicated by the presence of optical morphology peculiarities such as tidal tails;

2. a "control" sample of 6 groups with no optical indicators of gravitationally-induced tidal interactions between the group members.

An extensive optical ( $R$ and $\mathrm{H} \alpha$ ) and near-infrared $(J, H$ and $K$ band) study has already been completed for this sample (Marcum 1994), providing a complementary data set to the $\mathrm{H}$ I radio observations. A multi-wavelength comparative analysis utilizing results from this Nançay H I data set is planned for a future paper.

The "interacting" group sample comprises 62 galaxies in 9 groups, of which 8 galaxies were not previously observed in H I and 4 were not detected. The "control" sample comprises 40 galaxies in 6 groups, of which 3 were not previously observed and 6 were not detected in the $21-\mathrm{cm}$ line. Basic properties of the program objects are presented in Table 1.

Listed in the 7 columns of Table 1 are (1) Geller \& Huchra (1983) group designation number; (2) total number of galaxy members in group; (3) right ascension and declination of the group, from Geller \& Huchra (1983), converted from epoch B1950.0 to J2000.0; (4) mean recession velocity in $\mathrm{km} \mathrm{s}^{-1}$ of the group members, using optical radial velocities from the LEDA database and corrected to the Galactic Standard of Rest, following de Vaucouleurs et al. (1991, hereafter RC3); (5) velocity dispersion of group, using mean optical radial velocities from the LEDA database; (6) adopted distance in Mpc, derived from the mean recession velocity of the group, corrected for Virgocentric infall, following LEDA, and using a Hubble constant of $75 \mathrm{~km} \mathrm{~s}^{-1} \mathrm{Mpc}^{-1}$; (7) total apparent $B$ magnitude of group members, from Geller \& Huchra (1983).

Tables 2 and 3 list basic optical properties of the target objects. The 9 columns of Tables 2 and 3 are: (1) GH83 group designation number; (2) galaxy identification; (3) right ascension and declination of the galaxy centroid, computed from data taken from RC3; (4) morphological type, from LEDA and NED; (5) apparent $B_{\mathrm{T}}$ magnitude, from LEDA; (6) galaxy isophotal $B$ band diameter at the $25 \mathrm{mag} \operatorname{arcsec}^{-2}$ level, from LEDA; (7) axial ratio in the $B$ band, from LEDA; (8) optical recessional velocity, from LEDA; (9) error in recessional velocity, from LEDA.

\section{Observations and data reduction}

During the period December 1997 - May 1999 we obtained 21-cm H I spectra for all sample group galaxies using the Nançay Decimetric Radio Telescope and autocorrelator spectrometer. The Nançay telescope is a meridian transit-type instrument with an effective collecting area of roughly $7000 \mathrm{~m}^{2}$ (equivalent to a 94-m diameter parabolic dish). Due to the elongated geometry of the telescope, at 21-cm it has a half-power beam width of $3.6 \mathrm{E}-\mathrm{W} \times 22^{\prime} \mathrm{N}-\mathrm{S}$ for the range of declinations covered in this work (see Matthews \& van Driel 2000). Tracking was generally limited to about 45 min per source per day. Typical system temperatures were $\sim 40 \mathrm{~K}$. The Nançay beam is well suited for our project, since it will be sensitive to extended emission around group members which may have gone undetected with a smaller beam.

We obtained our observations in total power (positionswitching) mode using consecutive pairs of two-minute onand two-minute off-source integrations. Off-source integrations were taken at approximately $20^{\prime} \mathrm{E}$ of the target 
Table 2. Basic optical data for the interacting group sample.

\begin{tabular}{|c|c|c|c|c|c|c|c|c|c|c|}
\hline \multirow{2}{*}{$\begin{array}{l}\mathrm{GH} \\
\text { No. } \\
(1) \\
45\end{array}$} & \multirow{2}{*}{$\begin{array}{l}\text { Ident. } \\
(2) \\
\text { NGC } 2798^{*}\end{array}$} & \multicolumn{2}{|c|}{ 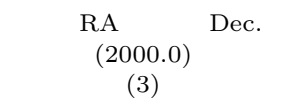 } & \multicolumn{2}{|c|}{$\begin{array}{l}\text { Morphol. Class. } \\
\begin{array}{ll}\text { LEDA NED } & (4)\end{array}\end{array}$} & \multirow{2}{*}{$\begin{array}{l}\begin{array}{l}B_{\mathrm{T}} \\
\mathrm{mag} \\
(5)\end{array} \\
13.03\end{array}$} & \multirow{2}{*}{$\begin{array}{l}D_{25} \\
\left({ }^{\prime}\right) \\
(6) \\
2.7\end{array}$} & \multirow{2}{*}{$\begin{array}{l}\text { axial } \\
\text { ratio } \\
(7)\end{array}$} & \multirow{2}{*}{$\begin{array}{r}V_{\mathrm{opt}} \\
\left(\mathrm{km} \mathrm{s}^{-1}\right) \\
(8) \\
1733\end{array}$} & \multirow{2}{*}{$\begin{array}{r}\text { err } \\
\left(\mathrm{km} \mathrm{s}^{-1}\right) \\
(9) \\
64\end{array}$} \\
\hline & & $\begin{array}{lll}09 & 17 & 22.9\end{array}$ & 420002 & $\mathrm{SBa}$ & SB(s)ap & & & & & \\
\hline & NGC $2799^{*}$ & $09 \quad 17 \quad 31.4$ & 415939 & $\mathrm{SBm}$ & $\mathrm{SB}(\mathrm{s}) \mathrm{m} ?$ & 13.96 & 1.8 & 0.29 & 1863 & 72 \\
\hline & NGC 2844 & 092148.0 & 400907 & $\mathrm{Sa}$ & SA(r)a: & 13.72 & 1.7 & 0.44 & 1495 & 16 \\
\hline & NGC 2852 & 092314.2 & $40 \quad 0953$ & $\mathrm{SBa}$ & $\mathrm{SAB}(\mathrm{r}) \mathrm{a} ?$ & 13.98 & 1.5 & 0.93 & 1821 & 54 \\
\hline \multirow[t]{10}{*}{58} & NGC 3162 & 101331.9 & 224423 & SBbc & $\mathrm{SAB}(\mathrm{rs}) \mathrm{bc}$ & 12.20 & 3.1 & 0.83 & 1456 & 56 \\
\hline & NGC 3177 & 101634.4 & 210729 & $\mathrm{Sb}$ & $\mathrm{SA}(\mathrm{rs}) \mathrm{b}$ & 13.01 & 1.5 & 0.76 & 1220 & 56 \\
\hline & NGC 3185 & 101738.6 & $2141 \quad 19$ & $\mathrm{SBa}$ & (R) SB(r)a & 12.82 & 1.7 & 0.6 & 1237 & 26 \\
\hline & NGC 3187 & $1017 \quad 47.5$ & $2152 \quad 25$ & $\mathrm{SBc}$ & SB(s)cp & 13.72 & 2.5 & 0.41 & 1582 & 28 \\
\hline & NGC 3189 & 101805.7 & 214959 & $\mathrm{Sa}$ & SA(s)ap & 11.86 & 4.6 & 0.42 & 1289 & 31 \\
\hline & NGC 3193 & 101825.0 & 215342 & $\mathrm{E}$ & E2 & 11.70 & 2.2 & 0.9 & 1378 & 27 \\
\hline & NGC 3213 & $\begin{array}{lll}10 & 21 & 17.7\end{array}$ & 193907 & $\mathrm{Sbc}$ & Sbc: & 14.13 & 1.0 & 0.79 & 1412 & 50 \\
\hline & NGC $3226 *$ & 102327.4 & 195355 & $\mathrm{E}$ & E2:p & 12.34 & 2.6 & 0.87 & 1325 & 72 \\
\hline & NGC $3227^{*}$ & 102331.4 & 195148 & SBa & $\mathrm{SAB}(\mathrm{s}) \mathrm{p}$ & 11.28 & 5.4 & 0.69 & 1145 & 54 \\
\hline & NGC 3239 & 102505.5 & 170935 & $\operatorname{Irr}$ & $\mathrm{IB}(\mathrm{s}) \mathrm{mp}$ & 11.71 & 4.5 & 0.54 & 830 & 52 \\
\hline \multirow[t]{8}{*}{67} & UGC 5870 & 104558.6 & 345753 & So & So? & 14.27 & 1.1 & 1.00 & 2032 & 50 \\
\hline & NGC 3381 & 104825.0 & 344244 & $\mathrm{SBb}$ & SBp & 12.77 & 2.0 & 0.92 & 1506 & 33 \\
\hline & NGC $3395^{*}$ & 104949.4 & 325851 & $\mathrm{SBc}$ & $\mathrm{SAB}(\mathrm{rs}) \mathrm{cdp}$ & 12.39 & 1.7 & 0.56 & 1634 & 39 \\
\hline & NGC $3396^{*}$ & 104956.1 & 325922 & $\mathrm{SBm}$ & IBmp & 12.48 & 2.7 & 0.42 & 1666 & 36 \\
\hline & NGC 3424 & 105146.6 & 325402 & $\mathrm{SBb}$ & $\mathrm{SB}(\mathrm{s}) \mathrm{b}: ?$ & 13.07 & 2.7 & 0.28 & 1420 & 35 \\
\hline & NGC 3430 & 105210.9 & 325709 & $\mathrm{SBc}$ & $\mathrm{SAB}(\mathrm{rs}) \mathrm{c}$ & 12.21 & 4.1 & 0.55 & 1577 & 77 \\
\hline & NGC 3442 & 105308.2 & 335436 & $\mathrm{Sa}$ & $\mathrm{Sa}$ ? & 13.80 & 0.6 & 0.77 & 1713 & 52 \\
\hline & UGC 6070 & 105946.5 & $\begin{array}{lll}33 & 23 & 32\end{array}$ & $\mathrm{Sm}$ & S? & 13.45 & 0.7 & 0.77 & 1861 & 60 \\
\hline \multirow[t]{3}{*}{86} & UGC 6545 & 113344.5 & 323804 & $\mathrm{SBb}$ & $\mathrm{S} ?$ & 14.46 & 1.2 & 0.37 & 4278 & 1885 \\
\hline & NGC $3786^{*}$ & 113942.4 & 315435 & $\mathrm{SBa}$ & $\mathrm{SAB}(\mathrm{rs}) \mathrm{ap}$ & 13.44 & 2.0 & 0.55 & 2596 & 296 \\
\hline & NGC $3788^{*}$ & 113944.0 & 315558 & SBab & $\mathrm{SAB}(\mathrm{rs}) \mathrm{abp}$ & 13.33 & 1.6 & 0.26 & 2486 & 150 \\
\hline 92 & NGC 3902 & 114918.9 & 260722 & $\mathrm{SBbc}$ & $\mathrm{SAB}(\mathrm{s}) \mathrm{bc}:$ & 13.76 & 1.6 & 0.81 & 3628 & 50 \\
\hline & NGC 3920 & 115006.3 & 245515 & & $\mathrm{~S}$ ? & 14.01 & 1.0 & 0.95 & 3611 & 37 \\
\hline & UGC 6806 & 115019.7 & 255742 & $\mathrm{Sc}$ & $\mathrm{Sp}$ & 14.17 & 1.9 & 0.32 & 3757 & 50 \\
\hline & NGC 3944 & 115305.5 & 261228 & E-S0 & S0-: & 14.12 & 1.4 & 0.77 & 3638 & 31 \\
\hline & IC 746 & 115534.6 & 255319 & $\mathrm{Sbc}$ & $\mathrm{Sb}$ & 14.36 & 1.1 & 0.30 & 5000 & 50 \\
\hline & NGC 3987 & $\begin{array}{lll}11 & 57 & 21.2\end{array}$ & 251141 & $\mathrm{Sb}$ & $\mathrm{Sb}$ & 13.90 & 2.2 & 0.19 & 4533 & 28 \\
\hline & NGC 3997 & 115747.3 & 251618 & $\mathrm{SBb}$ & SBbp & 14.02 & 1.6 & 0.55 & 4742 & 42 \\
\hline & NGC 4005 & 115810.3 & $25 \quad 07 \quad 18$ & $\mathrm{~S} ?$ & $\mathrm{Sb}$ & 13.89 & 1.2 & 0.59 & 4425 & 65 \\
\hline & NGC 4015A* & 115843.3 & 250240 & $\mathrm{Sc}$ & $\mathrm{E}$ & 14.15 & 0.9 & 0.23 & 4780 & 57 \\
\hline & NGC 4015B* & 115843.1 & 250235 & $\mathrm{Sab}$ & $\mathrm{S} ?$ & 12.81 & 1.4 & 0.66 & 4347 & 44 \\
\hline & NGC 4022 & 115901.1 & $25 \quad 13 \quad 19$ & So & $\operatorname{SAB} 0^{\circ}:$ & 14.24 & 1.3 & 0.95 & 4340 & 88 \\
\hline 126 & NGC 5341 & 135231.4 & 374858 & $\mathrm{SBd}$ & $\mathrm{S} ?$ & 14.08 & 1.3 & 0.42 & 3740 & 60 \\
\hline & NGC 5351 & 135328.1 & 375452 & $\mathrm{SBb}$ & $\mathrm{SA}(\mathrm{r}) \mathrm{b}:$ & 13.00 & 2.9 & 0.55 & 3845 & 66 \\
\hline & NGC 5378 & 135650.6 & 374800 & $\mathrm{SBa}$ & $\left(\mathrm{R}^{\prime}\right) \mathrm{SB}(\mathrm{r}) \mathrm{a}$ & 13.67 & 2.7 & 0.83 & 3017 & 42 \\
\hline & NGC 5380 & 135656.7 & 373634 & E-S0 & $\mathrm{SAO}^{-}$ & 13.26 & 1.9 & 1.00 & 3116 & 126 \\
\hline & NGC 5394* & 135833.8 & $3727 \quad 18$ & $\mathrm{SBb}$ & $\mathrm{SB}(\mathrm{s}) \mathrm{bp}$ & 13.69 & 1.7 & 0.66 & 3442 & 88 \\
\hline & NGC 5395* & 135838.3 & $\begin{array}{lll}37 & 25 & 32\end{array}$ & $\mathrm{Sb}$ & $\mathrm{SA}(\mathrm{s}) \mathrm{bp}$ & 12.12 & 2.6 & 0.53 & 3505 & 35 \\
\hline 141 & NGC 5529 & 141534.1 & 361336 & $\mathrm{Sc}$ & $\mathrm{Sc}$ & 12.74 & 6.5 & 0.11 & 2957 & 60 \\
\hline & NGC 5533 & 141607.6 & 352042 & $\mathrm{Sab}$ & $\mathrm{SA}(\mathrm{rs}) \mathrm{ab}$ & 12.70 & 3.2 & 0.60 & 3781 & 56 \\
\hline & NGC $5544^{*}$ & 141702.4 & 363421 & SO-a & (R)SB(rs) $0 / a$ & 13.97 & 1.1 & 0.89 & 3106 & 75 \\
\hline & NGC $5545^{*}$ & 141705.4 & 363434 & $\mathrm{Sbc}$ & SA(s)bc: & 14.90 & 1.0 & 0.34 & 3139 & 85 \\
\hline & NGC 5557 & 141826.2 & 362938 & $\mathrm{E}$ & E1 & 11.91 & 2.3 & 0.79 & 3221 & 43 \\
\hline & NGC 5589 & 142124.7 & 351615 & $\mathrm{SBa}$ & $\mathrm{SBa}$ & 14.20 & 1.1 & 1.00 & 3391 & 50 \\
\hline & NGC 5590 & 142138.0 & $\begin{array}{lll}35 & 12 \quad 19\end{array}$ & So & So & 13.42 & 1.8 & 1.0 & 3242 & 50 \\
\hline & NGC 5596 & 142229.2 & $3707 \quad 17$ & So & So & 14.41 & 1.1 & 0.74 & 3265 & 351 \\
\hline & NGC 5614 & 142408.2 & 345127 & Sab & $\mathrm{SA}(\mathrm{r}) \mathrm{abp}$ & 12.54 & 2.4 & 0.83 & 3872 & 41 \\
\hline & NGC 5656 & 143025.1 & $\begin{array}{lll}35 & 19 & 12\end{array}$ & $\mathrm{Sab}$ & $\mathrm{Sab}$ & 12.64 & 1.9 & 0.77 & 3150 & 9 \\
\hline & NGC 5675 & 143239.8 & $36 \quad 18 \quad 12$ & $\mathrm{SBb}$ & $\mathrm{S} ?$ & 13.72 & 2.8 & 0.35 & 4066 & 108 \\
\hline & NGC 5684 & 143549.8 & 363235 & So & So & 13.59 & 1.6 & 0.85 & 4082 & 31 \\
\hline & NGC 5695 & 143723.0 & 363415 & $\mathrm{SBa}$ & $\mathrm{SBb}$ & 13.58 & 1.5 & 0.7 & 4168 & 115 \\
\hline 153 & NGC 5929* & 152605.5 & 414017 & $\mathrm{Sa}$ & Sab:p & 14.06 & 1.0 & 0.95 & 2514 & 33 \\
\hline & NGC $5930^{*}$ & 152607.8 & 414039 & SBab & $\mathrm{SAB}(\mathrm{rs}) \mathrm{bp}$ & 13.53 & 1.8 & 0.46 & 2664 & 48 \\
\hline & UGC 9858 & 152640.9 & 403352 & SBbc & SABbc & 13.83 & 4.3 & 0.19 & 2624 & 8 \\
\hline 156 & NGC 5951 & 153343.1 & 150027 & $\mathrm{SBc}$ & SBc: & 13.47 & 3.6 & 0.22 & 1670 & 63 \\
\hline & NGC 5953* & 153432.4 & $15 \quad 1142$ & $\mathrm{~S} 0-\mathrm{a}$ & SAa:pec & 13.13 & 1.8 & 0.74 & 2061 & 90 \\
\hline & NGC 5954* & 153434.8 & $15 \quad 12 \quad 12$ & $\mathrm{SBc}$ & $\mathrm{SAB}(\mathrm{rs}) \mathrm{cd}: \mathrm{p}$ & 13.12 & 1.3 & 0.48 & 2034 & 81 \\
\hline & NGC 5962 & $\begin{array}{lll}15 & 36 & 31.7\end{array}$ & 163632 & $\mathrm{Sc}$ & $\mathrm{SA}(\mathrm{r}) \mathrm{c}$ & 12.03 & 3.0 & 0.72 & 1993 & 56 \\
\hline
\end{tabular}

Note: Asterisks $\left(^{*}\right)$ in this and following tables denote target pairs which are confused within a single Nançay beam (see Table 6, also). 
position. The autocorrelator was divided into two pairs of cross-polarized receiver banks, each with 512 channels and a $6.4 \mathrm{MHz}$ bandpass. This yielded a channel spacing of $2.64 \mathrm{~km} \mathrm{~s}^{-1}$, for an effective velocity resolution of $\sim 3.3 \mathrm{~km} \mathrm{~s}^{-1}$ at $21-\mathrm{cm}$, which was smoothed to a channel separation of 13.2 and a velocity resolution of $15.8 \mathrm{~km} \mathrm{~s}^{-1}$ during the data reduction, in order to search for faint features. The center frequencies of the two banks were tuned to the known redshifted $\mathrm{H}$ I frequency of the target. Total integration times were up to 5 hours per galaxy, depending on the strength of the source (see Tables 4 and 6).

We reduced our H I spectra using the standard DAC and SIR spectral line reduction packages available at the Nançay site. With this software we subtracted baselines (generally third order polynomials) and averaged the two receiver polarizations. To convert from units of $T_{\text {sys }}$ to flux density in mJy we used the calibration procedure described in Matthews et al. (2000), see also Matthews et al. (1998) and Matthews \& van Driel (2000). This procedure yields an internal calibration accuracy of about $\pm 15 \%$ near the rest frequency of the $21 \mathrm{~cm}$ line, where the observations presented here were made.

\section{Results}

Our reduced Nançay H I spectra are shown in Fig. 1. Integrated line fluxes, $I_{\mathrm{HI}}$, velocity widths at $50 \%$ and $20 \%$ of peak maximum, $W_{50}$ and $W_{20}$, radial velocities, $V_{\mathrm{HI}}$ and rms noise levels of our new spectra were measured using standard Nançay reduction software for galaxy observations.

Derived Nançay H I profile parameters for the target galaxies are listed in Tables 4 and 6. For each group of galaxies, a single distance was assumed (see Table 1), calculated using a Hubble constant of $75 \mathrm{~km} \mathrm{~s}^{-1} \mathrm{Mpc}^{-1}$ and the mean radial velocities of the group members, corrected for Virgocentric infall. No corrections have been applied to these values for, e.g., instrumental resolution or cosmological stretching (e.g., Matthews et al. 2000).

Listed in the 9 columns of Tables 4 and 6 are: (1) GH83 group designation number; (2) galaxy identification; (3) right ascension and declination of the galaxy centroid taken from the RC3; (4) recessional velocity, as derived from Nançay observations and corrected to the Galactic standard of rest, as well as its estimated uncertainty; (5) integrated neutral hydrogen line flux; (6) neutral hydrogen line full width measured at the $50 \%$ level of the peak flux density; (7) neutral hydrogen line full width measured at the $20 \%$ level of the peak flux density; (8) root-mean-square of the flux density, measured outside the emission line profile; (9) neutral hydrogen mass, using the adopted distance as given in Table 1; (10) luminosity in the $B$ band, using the adopted distance as given in Table 1 ; (11) neutral hydrogen mass to $B$ band luminosity ratio.

We estimated the uncertainties, $\sigma_{V_{\mathrm{HI}}}$, in the central H I velocities following Fouqué et al. (1990):

$\sigma_{V_{\mathrm{HI}}}=4 R^{0.5} P_{W}^{0.5} X^{-1}\left[\mathrm{~km} \mathrm{~s}^{-1}\right]$ where $R$ is the instrumental resolution $\left(15.8 \mathrm{~km} \mathrm{~s}^{-1}\right)$, $P_{W}=\left(W_{20}-W_{50}\right) / 2\left[\mathrm{~km} \mathrm{~s}^{-1}\right]$ and $X$ is the signal-to-noise ratio of a spectrum, which we defined as the ratio of the peak flux density and the rms noise. According to Fouqué et al., the uncertainty in the linewidths is $2 \sigma_{V_{\mathrm{HI}}}$ for $W_{50}$ and $3 \sigma_{V_{\mathrm{H}}}$ for $W_{20}$. The upper limits to the integrated $\mathrm{H}$ I line fluxes, $I_{\mathrm{HI}}$, are $3 \sigma$ values for flat-topped profiles with a width of $300 \mathrm{~km} \mathrm{~s}^{-1}$, a representative value for the galaxies detected.

\subsection{Notes to individual galaxies}

We searched the vicinity of the target objects for nearby spiral galaxies which could possibly give rise to confusion in those Nançay H I profiles where line emission was detected. We used the online NED and LEDA databases, in an area of $5.5 \times 30^{\prime}(\alpha \times \delta)$ about the pointing centre, i.e. about 1.5 times the HPBW, as well as optical images of a $24^{\prime} \times 24^{\prime}$ area around each target galaxy extracted from the Digitized Sky Survey.

We have summarized previously-published results of single-dish H I observations as well as of interferometric $\mathrm{H}$ I line imaging of galaxies and groups from the sample (see also the catalogue of H I maps by Martin 1998). Global profile parameters derived from these data are given in Tables 6 and 7, where the following columns are listed, while the keys to the telescopes and references used in these two tables are given in Table 8:

(1) GH83 group designation number; (2) galaxy identification; (3) mean velocity of the $\mathrm{H}$ I line profiles; (4) integrated line flux of the H I profiles; (5) H I line width measured at the $50 \%$ level of the peak flux density; (6) H I line width measured at the $20 \%$ level of the peak flux density; (7) telescope used (see Table 8); (8) literature reference (see Table 8).

For some objects, extended $\mathrm{H}$ I emission may have been missed by the single Nançay profile pointed towards the galaxy's centre. To assess which H I masses may be underestimated, we have assumed the $\mathrm{H}$ I diameters to be 1.25 times as large the optical $D_{25}$ dimensions, a rule-ofthumb from interferometric $\mathrm{H}$ I line imaging studies of normal, non-interacting spirals (e.g., Broeils \& van Woerden 1994, and references therein). We have also noted other points of interest, like the presence of active nuclei.

\subsubsection{Interacting group sample}

\section{Group GH 45:}

NGC 2798/9 pair: our Nançay profile, like all other published single-dish profiles of the pair, is undoubtedly confused by H I emission from nearby spiral UGC 4904. VLA H I observations (Nordgren et al. 1997; see Table 6) show an integrated line flux of $7.0 \mathrm{Jy} \mathrm{km} \mathrm{s}^{-1}$ and a mean velocity of about $1800 \mathrm{~km} \mathrm{~s}^{-1}$ for the pair, and $6.0 \mathrm{Jy} \mathrm{km} \mathrm{s}{ }^{-1}$ centered on $1670 \mathrm{~km} \mathrm{~s}^{-1}$ for UGC 4904. The pair shows an $\mathrm{HI}$ tail and signs of interaction in the velocity field; a total flux of about $5.0 \mathrm{Jy} \mathrm{km} \mathrm{s}^{-1}$ resides in the two disks, and about $2.0 \mathrm{Jy} \mathrm{km} \mathrm{s}^{-1}$ outside them. 
Table 3. Basic optical data for the control group sample.

\begin{tabular}{|c|c|c|c|c|c|c|c|c|c|c|}
\hline \multirow{4}{*}{$\begin{array}{l}\text { GH } \\
\text { No. } \\
(1) \\
49\end{array}$} & \multirow{2}{*}{$\begin{array}{l}\text { Ident. } \\
(2) \\
\text { NGC } 2998\end{array}$} & \multicolumn{2}{|c|}{$\begin{array}{l}\text { RA Dec. } \\
\underset{(2000.0)}{(3)}\end{array}$} & \multicolumn{2}{|c|}{$\begin{array}{l}\text { Morphol. Class. } \\
\begin{array}{l}\text { LEDA NED } \\
\end{array} \quad(4)\end{array}$} & \multirow{2}{*}{$\begin{array}{l}B_{\mathrm{T}} \\
\text { mag } \\
(5) \\
13.10\end{array}$} & \multirow{2}{*}{$\begin{array}{l}D_{25} \\
\left(\begin{array}{l}\prime \\
(6)\end{array}\right. \\
2.9\end{array}$} & \multirow{2}{*}{$\begin{array}{l}\text { axial } \\
\text { ratio } \\
(7)\end{array}$} & \multirow{2}{*}{$\begin{array}{r}V_{\mathrm{opt}} \\
\mathrm{km} \mathrm{s}^{-1} \\
(8) \\
4767\end{array}$} & \multirow{2}{*}{$\begin{array}{r}\text { err } \\
\mathrm{km} \mathrm{s}^{-1} \\
(9) \\
19\end{array}$} \\
\hline & & 094843.6 & 440452 & $\mathrm{SBc}$ & $\mathrm{SAB}(\mathrm{rs}) \mathrm{c}$ & & & & & \\
\hline & NGC 3009 & 095010.5 & 441740 & $\mathrm{Sc}$ & $\mathrm{S} ?$ & 13.36 & 0.8 & 0.93 & 4604 & 50 \\
\hline & UGC 5295 & 095254.1 & 425055 & $\mathrm{SBb}$ & $\mathrm{SAB}(\mathrm{s}) \mathrm{b}$ & 14.26 & 2.1 & 0.56 & 4805 & 40 \\
\hline \multirow[t]{4}{*}{57} & NGC 3156 & 101241.1 & 030750 & S0 & SO: & 13.07 & 1.9 & 0.62 & 1230 & 89 \\
\hline & NGC 3165 & 101331.4 & 032232 & $\mathrm{Sm}$ & $\mathrm{SA}(\mathrm{s}) \mathrm{dm}$ & 14.49 & 1.4 & 0.49 & 1317 & 50 \\
\hline & NGC 3166 & 101344.9 & 032531 & $\mathrm{~S} 0-\mathrm{a}$ & $\mathrm{SABO}^{-} \mathrm{a}$ & 11.22 & 4.7 & 0.46 & 1329 & 75 \\
\hline & NGC 3169 & 101414.3 & 032808 & $\mathrm{Sa}$ & $\mathrm{SA}(\mathrm{s}) \mathrm{ap}$ & 11.04 & 4.6 & 0.56 & 1253 & 46 \\
\hline \multirow[t]{7}{*}{89} & UGC 6617 & 113917.5 & 095748 & S0 & S0? & 14.28 & 0.9 & 0.36 & 6228 & 31 \\
\hline & NGC 3817 & 114153.1 & 101807 & S0-a & $\mathrm{SB} 0 / \mathrm{a}$ & 14.27 & 0.7 & 0.76 & 6102 & 32 \\
\hline & NGC $3822^{*}$ & 114211.3 & 101640 & S0 & $\mathrm{Sb}$ & 14.10 & 0.8 & 0.60 & 6132 & 33 \\
\hline & NGC $3825^{*}$ & 114223.7 & 101552 & $\mathrm{SBa}$ & $\mathrm{SBa}$ & 13.93 & 0.9 & 0.81 & 6436 & 132 \\
\hline & IC 724 & 114334.7 & 085631 & $\mathrm{Sa}$ & $\mathrm{Sa}$ & 13.49 & 2.3 & 0.40 & 5959 & 16 \\
\hline & NGC 3843 & 114354.1 & 075532 & $\mathrm{~S} 0-\mathrm{a}$ & $\mathrm{S} 0 / \mathrm{a}$ & 14.00 & 1.0 & 0.45 & 5908 & 50 \\
\hline & NGC 3839 & 114354.4 & 104659 & $\mathrm{Sd}$ & Sdm: & 13.60 & 1.0 & 0.51 & 5920 & 45 \\
\hline \multirow[t]{3}{*}{118} & NGC 5141 & 132451.6 & 362240 & S0 & S0 & 13.80 & 1.7 & 0.76 & 5201 & 101 \\
\hline & NGC 5142 & 132501.3 & 362358 & S0 & S0 & 14.20 & 1.3 & 0.67 & 5235 & 65 \\
\hline & NGC 5149 & 132609.7 & 355604 & $\mathrm{SBbc}$ & $\mathrm{SBbc}$ & 13.90 & 1.5 & 0.64 & 5601 & 49 \\
\hline \multirow[t]{17}{*}{123} & NGC 5289 & 134509.2 & 413011 & SBab & (R)SABab: & 13.95 & 1.6 & 0.31 & 2446 & 51 \\
\hline & NGC 5290 & 134519.2 & 414255 & $\mathrm{Sbc}$ & Sbc: & 13.29 & 3.7 & 0.27 & 2544 & 48 \\
\hline & NGC 5297 & 134624.1 & 435225 & $\mathrm{SBc}$ & $\mathrm{SAB}(\mathrm{s}) \mathrm{c}:$ & 12.39 & 5.5 & 0.23 & 2492 & 106 \\
\hline & NGC 5311 & 134855.9 & 395907 & $\mathrm{~S} 0-\mathrm{a}$ & $\mathrm{S} 0 / \mathrm{a}$ & 13.59 & 2.6 & 0.81 & 2698 & 40 \\
\hline & UGC 8736 & 134903.9 & 392956 & $\mathrm{Sc}$ & $\mathrm{S} ?$ & 14.25 & 1.3 & 0.42 & 2404 & 50 \\
\hline & NGC 5313 & 134944.7 & 395910 & $\mathrm{Sb}$ & Sb? & 12.71 & 1.7 & 0.56 & 2597 & 44 \\
\hline & NGC 5320 & 135020.5 & 412206 & $\mathrm{SBc}$ & $\mathrm{SAB}(\mathrm{rs}) \mathrm{c}:$ & 12.96 & 3.6 & 0.49 & 2646 & 65 \\
\hline & NGC 5326 & 135050.7 & 393429 & $\mathrm{Sa}$ & SAa: & 12.90 & 2.2 & 0.54 & 2540 & 68 \\
\hline & NGC 5336 & 135210.9 & 431434 & $\mathrm{Sc}$ & Scd: & 13.57 & 1.4 & 0.89 & 2297 & 60 \\
\hline & NGC 5337 & 135223.1 & 394115 & $\mathrm{~S} ?$ & $\mathrm{~S} ?$ & 13.35 & 1.7 & 0.48 & 2208 & 46 \\
\hline & NGC 5350* & 135321.5 & 402148 & $\mathrm{SBbc}$ & $\mathrm{SB}(\mathrm{r}) \mathrm{b}$ & 12.23 & 2.7 & 0.80 & 2322 & 61 \\
\hline & NGC $5354^{*}$ & 135326.6 & 401816 & S0 & $\mathrm{E} 2$ & 12.40 & 1.7 & 0.87 & 2681 & 244 \\
\hline & NGC 5353 & 135326.8 & 401703 & S0 & S0 & 11.97 & 1.9 & 0.71 & 2170 & 103 \\
\hline & NGC 5355 & 135345.9 & 402017 & S0 & E3 & 13.98 & 0.6 & 0.64 & 2422 & 38 \\
\hline & NGC 5362 & 135453.5 & 411851 & $\mathrm{Sb}$ & $\mathrm{Sb} ? \mathrm{p}$ & 13.20 & 2.2 & 0.44 & 2228 & 45 \\
\hline & NGC 5371 & 135540.5 & 402744 & $\mathrm{SBbc}$ & $\mathrm{SAB}(\mathrm{rs}) \mathrm{bc}$ & 11.38 & 4.2 & 0.81 & 2570 & 40 \\
\hline & NGC 5383 & 135705.2 & 415044 & $\mathrm{SBb}$ & $\left(\mathrm{R}^{\prime}\right) \mathrm{SB}(\mathrm{rs}) \mathrm{b}: \mathrm{p}$ & 12.10 & 2.7 & 0.81 & 2227 & 26 \\
\hline \multirow[t]{6}{*}{155} & NGC 5934 & 152812.4 & 425549 & $\mathrm{~S} ?$ & $\mathrm{~S} ?$ & 14.60 & 0.7 & 0.47 & 5600 & 39 \\
\hline & NGC 5945 & 152945.2 & 425514 & SBab & $\mathrm{SB}(\mathrm{rs}) \mathrm{ab}$ & 13.80 & 2.6 & 0.85 & 5521 & 50 \\
\hline & IC 4562 & 153557.6 & 432940 & $\mathrm{E}$ & $\mathrm{E} ?$ & 13.81 & 1.2 & 1.00 & 5666 & 145 \\
\hline & IC $4564^{*}$ & 153627.0 & 433108 & $\mathrm{Sc}$ & $\mathrm{S} ?$ & 14.41 & 1.3 & 0.35 & 5669 & 39 \\
\hline & IC $4566^{*}$ & 153642.6 & 433224 & $\mathrm{Sab}$ & $\mathrm{Sab}$ & 14.11 & 1.6 & 0.64 & 5608 & 47 \\
\hline & IC 4567 & 153713.3 & 431754 & $\mathrm{Sc}$ & Scd? & 13.51 & 1.4 & 0.71 & 5775 & 40 \\
\hline
\end{tabular}

Note: Asterisks $(*)$ in this and following tables denote target pairs which are confused within a single Nançay beam (see Table 7 , also).

\section{Group GH 58:}

$N G C$ 3162: Four of the six available integrated line fluxes of this object are in agreement $\left(\sim 26 \mathrm{Jy} \mathrm{km} \mathrm{s}^{-1}\right)$, the exceptions being the considerably higher Nançay value (44.1 $\mathrm{Jy} \mathrm{km} \mathrm{s}{ }^{-1}$ ) of Bottinelli et al. (1982) and the considerably lower (6.2 $\mathrm{Jy} \mathrm{km} \mathrm{s}^{-1}$ ) Arecibo value of Williams \& Rood (1987), whose $W_{20}$ of $605 \mathrm{~km} \mathrm{~s}^{-1}$ is about three times larger than the other available values.

$N G C$ 3177: The 2 Nançay and the 3 Arecibo $I_{\mathrm{HI}}$ measurements are about equally divided around two values 


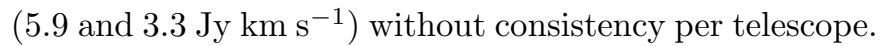
This excludes a possible beam size effect and, in any case, the $\mathrm{H}$ I diameter of this galaxy with its $1.5 D_{25}$ diameter is not expected to exceed the beam size of either telescope.

NGC 3185/87/89/93 subgroup: the area covering NGC $3185 / 87 / 89 / 93$ was mapped in $\mathrm{HI}$ at the VLA by Williams et al. (1991), who, erroneously, refer to NGC 3189 as NGC 3190. These data show that our NGC 3185 spectrum will be confused by emission from NGC 3187 in the $\sim 1300-1400 \mathrm{~km} \mathrm{~s}^{-1}$ range, and that our NGC 3187 and NGC 3189 spectra will be confused by each other in the $\sim 1400-1500 \mathrm{~km} \mathrm{~s}^{-1}$ range. The Nançay pointings should cover essentially all emission from NGC 3187 and 3189, judging from the VLA H I column density map. The $\mathrm{H}$ I line flux we measured towards NGC 3187 (8.8 Jy $\mathrm{km} \mathrm{s}^{-1}$ ) is comparable to the value of $10.6 \mathrm{Jy} \mathrm{km} \mathrm{s}{ }^{-1}$ measured at the VLA for this object, though on average two times lower than the previously published Nançay values (24.6: and 14.2 from, respectively, Bottinelli et al. 1982; Balkowski \& Chamaraux 1983). The uncertain integrated H I line flux of NGC 3189 measured at Green Bank (7.3: Jy km s${ }^{-1}$, Huchtmeier 1982) and the Arecibo measurement of 6.1 by Krumm \& Salpeter (1982) are about twice as large as our Nançay value (3.1). Our value is consistent, however, with the mean of the four other literature values, $3.5 \mathrm{Jy} \mathrm{km} \mathrm{s}^{-1}$. NGC 3185 is an (R)SB(r)a with a Seyfert2 spectrum, and NGC 3189 an SA(s)apec with a LINER spectrum.

NGC 3226/7 pair: mapped in $\mathrm{H}$ I at the VLA (Mundell et al. 1995). No H I was detected in NGC 3226, and NGC 3227 shows a complex H I distribution and kinematics. About half the $\mathrm{H}$ I resides in a disk with a normal rotation curve, the rest in two plumes extending $7^{\prime} \mathrm{N}$ and $16^{\prime} \mathrm{S}$ of the system, and in an $\mathrm{H}$ I feature at the base of the $\mathrm{N}$ plume which may be a gas-rich dwarf galaxy. As the plumes have a N-S orientation, the Nançay beam will cover almost all of the $\mathrm{H}$ I emission, as the southernmost half of the southern plume is relatively faint compared to the bulk of the emission. Our Nançay integrated line flux (16.6 $\mathrm{Jy} \mathrm{km} \mathrm{s}^{-1}$ ) is consistent with the Green Bank and Jodrell Bank data, while the Arecibo values are a bit lower. The only significantly discrepant Arecibo value is the $6.8 \mathrm{Jy} \mathrm{km} \mathrm{s}^{-1}$ from Chamaraux et al. (1987). Our $W_{20}$ profile width is consistent with the other measurements, but our $W_{50}$ value $\left(103 \mathrm{~km} \mathrm{~s}^{-1}\right)$ is similar to the Green Bank measurement by Peterson (1979) only, the 5 other published values are $\sim 350 \mathrm{~km} \mathrm{~s}^{-1}$ on average. NGC 3226 is an E2:pec with a LINER spectrum, and NGC 3227 an $\mathrm{SAB}(\mathrm{s})$ pec with a Seyfert1.5 spectrum.

NGC 3239: Four small galaxies will be included in the Nançay beam centered on this nearby $\left(V=830 \mathrm{~km} \mathrm{~s}^{-1}\right)$ irregular galaxy. Three of these have been previously catalogued, two (CGCG 094-039 and CGCG 094-043) with optical redshifts (Falco et al. 1999) around 13,300 $\mathrm{km} \mathrm{s}^{-1}$, and another, CGCG 094-040, without known redshift. The latter, faint $\left(B_{\mathrm{T}} 15.3 \mathrm{mag}\right)$ object of less than $1^{\prime}$ diameter, is not expected to cause confusion with the very strong (75 $\mathrm{Jy} \mathrm{km} \mathrm{s}^{-1}$ ) H I detection of NGC 3239.
The nearby companions may well explain the much higher H I line flux measured at Green Bank ( 80 Jy $\mathrm{km} \mathrm{s}^{-1}$ on average), compared to the Nançay and Arecibo values (43-61 Jy $\mathrm{km} \mathrm{s}^{-1}$ ) - see Table 6. In principle, some H I emission from NGC 3239 may fall outside the Nançay beam, as its optical E-W $D_{25}$ diameter of 4.5 exceeds the 3'6 E-W HPBW, and as it looks like the result of a recent merger, which might have $\mathrm{H}$ I plumes associated with it, not covered by the Nançay beam.

\section{Group GH 67:}

$U G C$ 5870: we registered an off-band detection of a galaxy at $V=1616 \mathrm{~km} \mathrm{~s}^{-1}$, with a $F W H M=$ $120 \mathrm{~km} \mathrm{~s}^{-1}$. This detection does not appear to disturb the detection of the target galaxy, which shows a classical double-horned spectrum.

$N G C$ 3381: The integrated line flux of $21 \mathrm{Jy} \mathrm{km} \mathrm{s}^{-1}$ measured at Arecibo by Krumm \& Salpeter (1980) is the result of a crude mapping of the galaxy's H I distribution; a lower limit of $10^{\prime}$ is given by the authors for the $\mathrm{H}$ I diameter, indicating that the actual extent exceeds the mapped area. The other available $I_{\mathrm{HI}}$ values $\left(8.5-13 \mathrm{Jy} \mathrm{km} \mathrm{s}^{-1}\right)$ underestimate the total line flux. No galaxies that could be potential sources of confusion were found in a $24^{\prime} \times 24^{\prime}$ area centered on the object.

$N G C$ 3395/96: this pair was mapped in $\mathrm{H}$ I at the VLA (Clemens et al. 1999). An integrated flux of $22 \mathrm{Jy} \mathrm{km} \mathrm{s}^{-1}$ was detected in the pair, which shows a clear H I bridge, as well as $4 \mathrm{Jy} \mathrm{km} \mathrm{s}^{-1}$ in an $\mathrm{H}$ I tail extending to a distance of $10^{\prime} \mathrm{SW}$ of the pair. The H I kinematics were modeled by the authors using $N$-body simulations, which indicate that the tail was stripped from NGC 3395. The Nançay and Arecibo line fluxes agree well, and are about two times smaller than the $38.4 \mathrm{Jy} \mathrm{km} \mathrm{s}^{-1}$ was at Green Bank by Shostak (1975).

$N G C 3424$ and NGC 3430: our Nançay profiles of these two nearby objects ( $4.5 \mathrm{E}-\mathrm{W}$ separation) will be mutually confused by their line emission, as will the published Green Bank and Jodrell Bank profiles of NGC 3430. The Arecibo profiles (Helou et al. 1982) of the two galaxies are not expected to be confused, given the telescope's small HPBW. The Arecibo central velocities are $1501 / 1586 \mathrm{~km} \mathrm{~s}^{-1}$, the $F W H M \mathrm{~s} 353 / 340 \mathrm{~km} \mathrm{~s}^{-1}$, and the integrated line fluxes 14.0/44.1 $\mathrm{Jy} \mathrm{km} \mathrm{s}^{-1}$, respectively.

NGC 3442: our Nançay profile parameters agree well with those of 4 of the 5 available Arecibo profiles; the integrated line flux measured at Arecibo by Magri (1994) is about 3 times larger (9.1 Jy $\mathrm{km} \mathrm{s}^{-1}$ ), though the profile's central velocity and linewidth are consistent the other measurements.

\section{Group GH 86:}

$U G C$ 6545: for this object two completely discrepant optical redshifts have been published: $2619 \pm 41 \mathrm{~km} \mathrm{~s}^{-1}$, a CfA redshift value (Huchra et al. 1983; Huchra et al. 1995) and $6419 \pm 90 \mathrm{~km} \mathrm{~s}^{-1}$ (Karachentsev 1980). Our Nançay value, $2630 \mathrm{~km} \mathrm{~s}^{-1}$, is consistent with the Huchra et al. value. 
Table 4. Nançay H I line data for the interacting group sample.

\begin{tabular}{|c|c|c|c|c|c|c|c|c|c|c|c|}
\hline No. & Ident. & RA & Dec. & $\mathrm{km} \mathrm{s}^{-1}$ & $\mathrm{Jy} \mathrm{km} \mathrm{s}^{-1}$ & $\mathrm{~km} \mathrm{~s}^{-1}$ & $\mathrm{~km} \mathrm{~s}^{-1}$ & mJy & $\begin{array}{c}M_{\mathrm{HI}} \\
{[\log ]} \\
M_{\odot}\end{array}$ & $\begin{array}{r}L_{B} \\
{[\log ]} \\
L_{\odot, B}\end{array}$ & $\begin{array}{l}M_{\mathrm{HI}} / L_{B} \\
M_{\odot} / L_{\odot, B}\end{array}$ \\
\hline \multirow[t]{3}{*}{45} & $\mathrm{~N} 2798 / 9^{*}$ & 091727 & 415950 & $1750 \pm 8$ & 10.9 & 304 & 424 & 2.7 & 9.18 & 9.90 & 0.19 \\
\hline & N2844 & 092148 & $\begin{array}{lll}40 & 0907\end{array}$ & $1494 \pm 4$ & 7.6 & 309 & 337 & 2.8 & 9.03 & 9.48 & 0.35 \\
\hline & N2852 & 092314 & 400953 & $1781 \pm 3$ & 5.2 & 211 & 232 & 2.1 & 8.86 & 9.37 & 0.31 \\
\hline \multirow[t]{9}{*}{58} & N3162 & 101332 & 224423 & $1301 \pm 2$ & 25.6 & 183 & 204 & 5.9 & 9.27 & 9.80 & 0.30 \\
\hline & N3177 & 101635 & 210729 & $1326 \pm 16$ & 3.4 & 180 & 325 & 3.3 & 8.39 & 9.48 & 0.08 \\
\hline & N3185 & 101739 & 214119 & $1225 \pm 6$ & 3.1 & 248 & 276 & 2.5 & 8.35 & 9.55 & 0.06 \\
\hline & N3187 & 101748 & 215225 & $1589 \pm 11$ & 8.8 & 236 & 296 & 5.5 & 8.81 & 9.19 & 0.41 \\
\hline & N3189 & 101806 & 214953 & $1300 \pm 23$ & 5.0 & 458 & 560 & 4.4 & 8.56 & 9.94 & 0.04 \\
\hline & N3193 & 101825 & 215342 & $1361 \pm 25$ & 1.5 & 470 & 514 & 2.2 & 8.05 & 10.00 & 0.01 \\
\hline & N3213 & 102118 & 193907 & $1345 \pm 13$ & 1.3 & 149 & 187 & 2.4 & 7.97 & 9.03 & 0.09 \\
\hline & $\mathrm{N} 3226 / 7^{*}$ & 102330 & 195251 & $1146 \pm 5$ & 16.6 & 103 & 437 & 2.3 & 9.08 & 10.31 & 0.06 \\
\hline & N3239 & 102506 & 170935 & $762 \pm 1$ & 43.3 & 132 & 183 & 3.5 & 9.50 & 10.00 & 0.32 \\
\hline \multirow[t]{7}{*}{67} & U5870 & 104559 & 345753 & $1992 \pm 4$ & 4.6 & 229 & 244 & 2.9 & 8.80 & 9.25 & 0.35 \\
\hline & N3381 & 104825 & 344244 & $1629 \pm 2$ & 13.0 & 68 & 135 & 3.5 & 9.25 & 9.85 & 0.25 \\
\hline & N3395/6* & 104953 & 325906 & $1637 \pm 2$ & 18.1 & 105 & 180 & 2.6 & 9.40 & 10.29 & 0.13 \\
\hline & N3424 & 105147 & 325402 & $1507 \pm 5$ & 15.5 & 318 & 401 & 2.8 & 9.33 & 9.73 & 0.40 \\
\hline & N3430 & 105211 & 325709 & $1584 \pm 2$ & 30.7 & 333 & 353 & 3.9 & 9.63 & 10.07 & 0.36 \\
\hline & N3442 & 105308 & 335436 & $1731 \pm 5$ & 3.2 & 133 & 160 & 2.1 & 8.64 & 9.44 & 0.16 \\
\hline & U6070 & 105947 & 332332 & $1853 \pm 5$ & 5.2 & 115 & 157 & 2.9 & 8.85 & 9.58 & 0.19 \\
\hline \multirow[t]{2}{*}{86} & U6545 & 113345 & 323804 & 2630 & 1.0 & 150: & & 2.5 & 8.53 & 9.55 & 0.10 \\
\hline & N3786/8* & 113943 & 315516 & $2674 \pm 7$ & 14.1 & 436 & 535 & 2.3 & 9.57 & 10.28 & 0.19 \\
\hline \multirow[t]{10}{*}{92} & N3902 & 114919 & 260722 & $3651 \pm 11$ & 8.9 & 241 & 371 & 4.5 & 9.85 & 10.22 & 0.43 \\
\hline & N3920 & 115006 & 245515 & $3635 \pm 3$ & 8.4 & 181 & 211 & 2.9 & 9.82 & 10.12 & 0.51 \\
\hline & U6806 & 115020 & 255742 & $3751 \pm 2$ & 17.5 & 205 & 245 & 2.8 & 10.14 & 10.05 & 1.24 \\
\hline & N3944 & 115306 & 261228 & - & $<3.1$ & - & - & 3.4 & $<9.39$ & 10.07 & $<0.21$ \\
\hline & I746 & 115535 & 255319 & $5027 \pm 6$ & 6.7 & 268 & 288 & 3.9 & 9.73 & 9.97 & 0.57 \\
\hline & N3987 & 115721 & 251141 & $4450 \pm 7$ & 7.8 & 521 & 551 & 2.3 & 9.79 & 10.16 & 0.43 \\
\hline & N3997 & 115747 & 251618 & $4771 \pm 5$ & 7.6 & 241 & 273 & 3.2 & 9.78 & 10.11 & 0.47 \\
\hline & N4005 & 115810 & 250718 & - & $<2.3$ & - & - & 2.6 & $<9.27$ & 10.16 & $<0.13$ \\
\hline & N4015A/B* & 115843 & 250235 & $4516 \pm 25$ & 3.1 & 404 & 524 & 3.1 & 9.39 & 10.70 & 0.05 \\
\hline & N4022 & 115901 & 251319 & - & $<2.6$ & - & - & 2.9 & $<9.34$ & 10.02 & $<0.20$ \\
\hline \multirow[t]{5}{*}{126} & N5341 & 135232 & 374858 & $3649 \pm 4$ & 5.1 & 252 & 265 & 3.1 & 9.42 & 9.91 & 0.33 \\
\hline & N5351 & 135328 & 375452 & $3607 \pm 4$ & 18.3 & 415 & 438 & 5.1 & 9.98 & 10.34 & 0.44 \\
\hline & N5378 & 135651 & 374800 & $3000 \pm 22$ & 3.9 & 301 & 367 & 5.0 & 9.31 & 10.07 & 0.17 \\
\hline & N5380 & 135657 & 373634 & $3000 \pm 11$ & 2.9 & 304 & 336 & 2.5 & 9.18 & 10.23 & 0.09 \\
\hline & N5394/5* & 135836 & 372625 & $3454 \pm 12$ & 20.0 & 457 & 577 & 5.4 & 10.02 & 10.78 & 0.17 \\
\hline \multirow[t]{12}{*}{141} & N5529 & 141534 & 361336 & $2884 \pm 3$ & 26.4 & 565 & 604 & 3.1 & 10.17 & 10.49 & 0.48 \\
\hline & N5533 & 141608 & $35 \quad 2042$ & $3864 \pm 2$ & 19.3 & 431 & 456 & 2.7 & 10.04 & 10.49 & 0.35 \\
\hline & N5544/5* & 141704 & 363428 & $3071 \pm 14$ & 3.8 & 247 & 310 & 2.9 & 9.33 & 10.14 & 0.16 \\
\hline & N5557 & 141826 & 362938 & - & $<2.6$ & - & - & 2.9 & $<9.17$ & 10.81 & $<0.02$ \\
\hline & N5589 & 142125 & $\begin{array}{llll}35 & 16 & 15\end{array}$ & $3397 \pm 8$ & 1.3 & 182 & 195 & 2.2 & $<8.20$ & 9.89 & 0.10 \\
\hline & N5590 & 142138 & $\begin{array}{lll}35 & 12 & 19\end{array}$ & - & $<2.8$ & - & - & 3.1 & $<8.96$ & 10.21 & $<0.10$ \\
\hline & N5596 & 142229 & $\begin{array}{lll}37 & 07 & 17\end{array}$ & $3122 \pm 13$ & 1.0 & 544 & 554 & 3.4 & 8.75 & 9.81 & 0.09 \\
\hline & N5614 & 142408 & 345127 & $3893 \pm 12$ & 3.2 & 138 & 246 & 2.3 & 9.26 & 10.56 & 0.05 \\
\hline & N5656 & 143025 & 351912 & $3163 \pm 6$ & 8.2 & 338 & 377 & 3.2 & 9.67 & 10.52 & 0.14 \\
\hline & N5675 & 143240 & 361812 & - & $<2.2$ & - & - & 2.4 & $<9.09$ & 10.09 & $<0.10$ \\
\hline & N5684 & 143550 & 363235 & - & $<2.3$ & - & - & 2.6 & $<9.12$ & 10.14 & $<0.10$ \\
\hline & N5695 & 143723 & 363415 & - & $<2.4$ & - & - & 2.7 & $<9.13$ & 10.14 & $<0.10$ \\
\hline \multirow[t]{2}{*}{153} & N5929/30* & 152607 & 414028 & $2539 \pm 14$ & 3.0 & 219 & 299 & 2.3 & 8.96 & 10.13 & 0.07 \\
\hline & U9858 & 152641 & 403352 & $2621 \pm 2$ & 30.5 & 364 & 388 & 2.6 & 10.00 & 9.80 & 1.57 \\
\hline \multirow[t]{3}{*}{156} & N5951 & 153343 & 150027 & $1780 \pm 1$ & 17.5 & 265 & 284 & 1.6 & 9.48 & 9.67 & 0.65 \\
\hline & N5953/4* & 153433 & 151157 & $1966 \pm 4$ & 7.7 & 146 & 275 & 1.9 & 9.13 & 9.81 & 0.21 \\
\hline & N5962 & 153632 & 163632 & $1957 \pm 2$ & 17.6 & 341 & 364 & 2.3 & 9.49 & 10.25 & 0.17 \\
\hline
\end{tabular}

NGC 3786/8: short Westerbork synthesis observations of the pair were obtained by Oosterloo \& Shostak (1993), but they refrain from listing global line parameters as NGC 3786 (= UGC 6621) is "not detected or confused with NGC 3788 (= UGC 6623)", and NGC 3788 is "possibly confused with NGC 3786". Their Arecibo observations of the pair were confused as well and no single-dish profile parameters are given. Our Nançay integrated line flux (14.1 $\mathrm{Jy} \mathrm{km} \mathrm{s}^{-1}$ ) is similar to the Green Bank value, and both are about 1.5 times larger than 4 of the 5 available Arecibo values, the exception being the much lower (4.9 $\mathrm{Jy} \mathrm{km} \mathrm{s}^{-1}$ ) measurement by
Lewis et al. (1985). NGC 3786 is an SAB(rs)apec with a Seyfert1.8 spectrum.

\section{Group GH 92:}

$U G C$ 6806: our Nançay profile will be confused by nearby UGC 6807, a $B_{\mathrm{T}} 15.0 \mathrm{mag}$ Irregular at $2^{\prime}$ separation. Even the Arecibo profile of UGC 6806 (Williams 1986, see Table 6) should be confused, as their HPBW is about the same as the galaxies' separation. The Nançay integrated line flux (17.5 $\mathrm{Jy} \mathrm{km} \mathrm{s}^{-1}$ ) is 4 times higher than the Arecibo value, though the central velocities and profile widths are quite similar. For UGC 6807, only a Nançay 

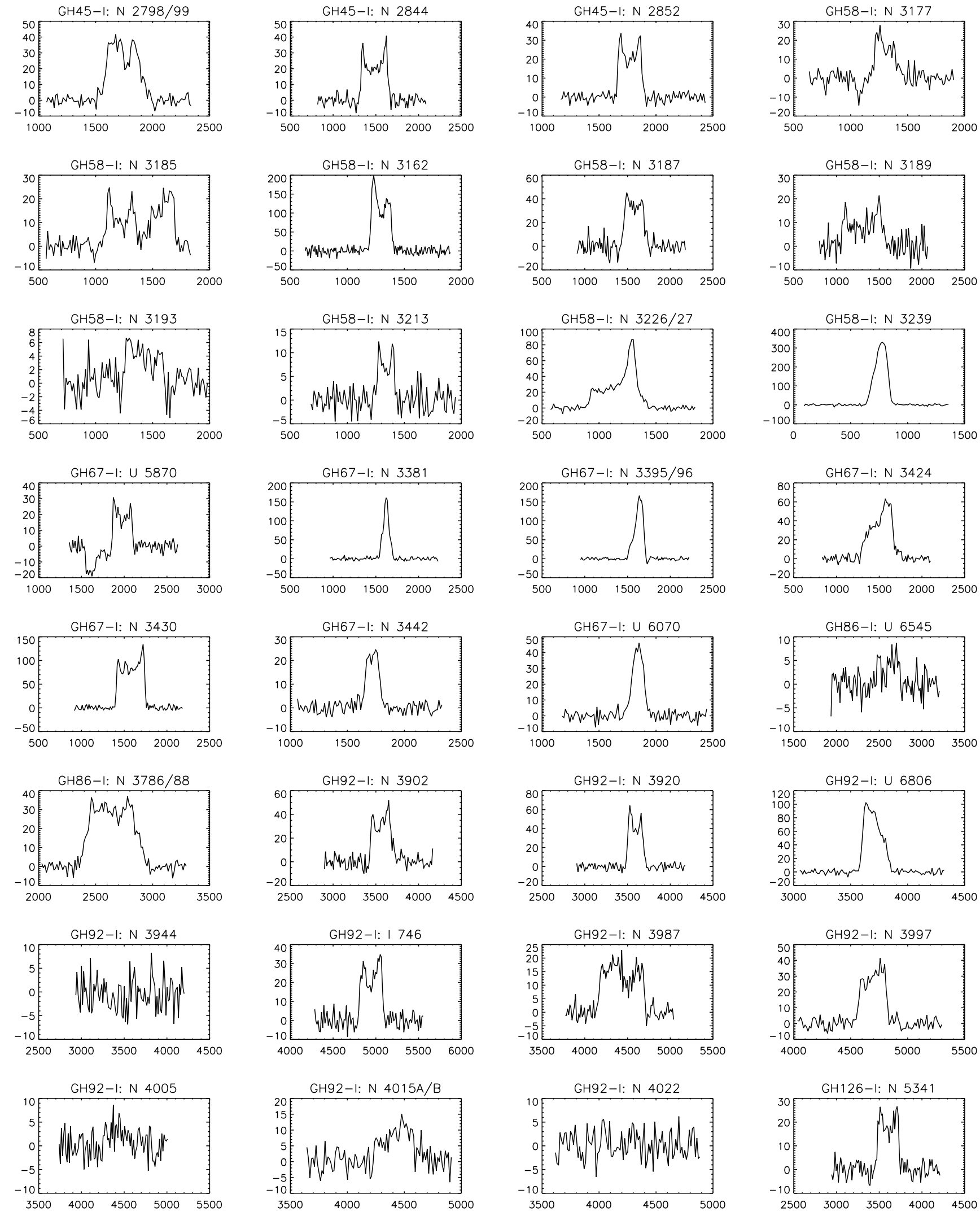

Fig. 1. a) Nançay 21-cm H I line spectra. Each galaxy is identified by its GH group number, followed by an "I" for the interacting sample or a "C" for the control sample, and its individual catalog identification. The velocity resolution of the spectra is $15.8 \mathrm{~km} \mathrm{~s}^{-1}$. The axes are heliocentric velocity (in $\mathrm{km} \mathrm{s}^{-1}$ ) and flux density (in $\mathrm{mJy}$ ). 
W. van Driel et al.: H I observations of loose galaxy groups. I.
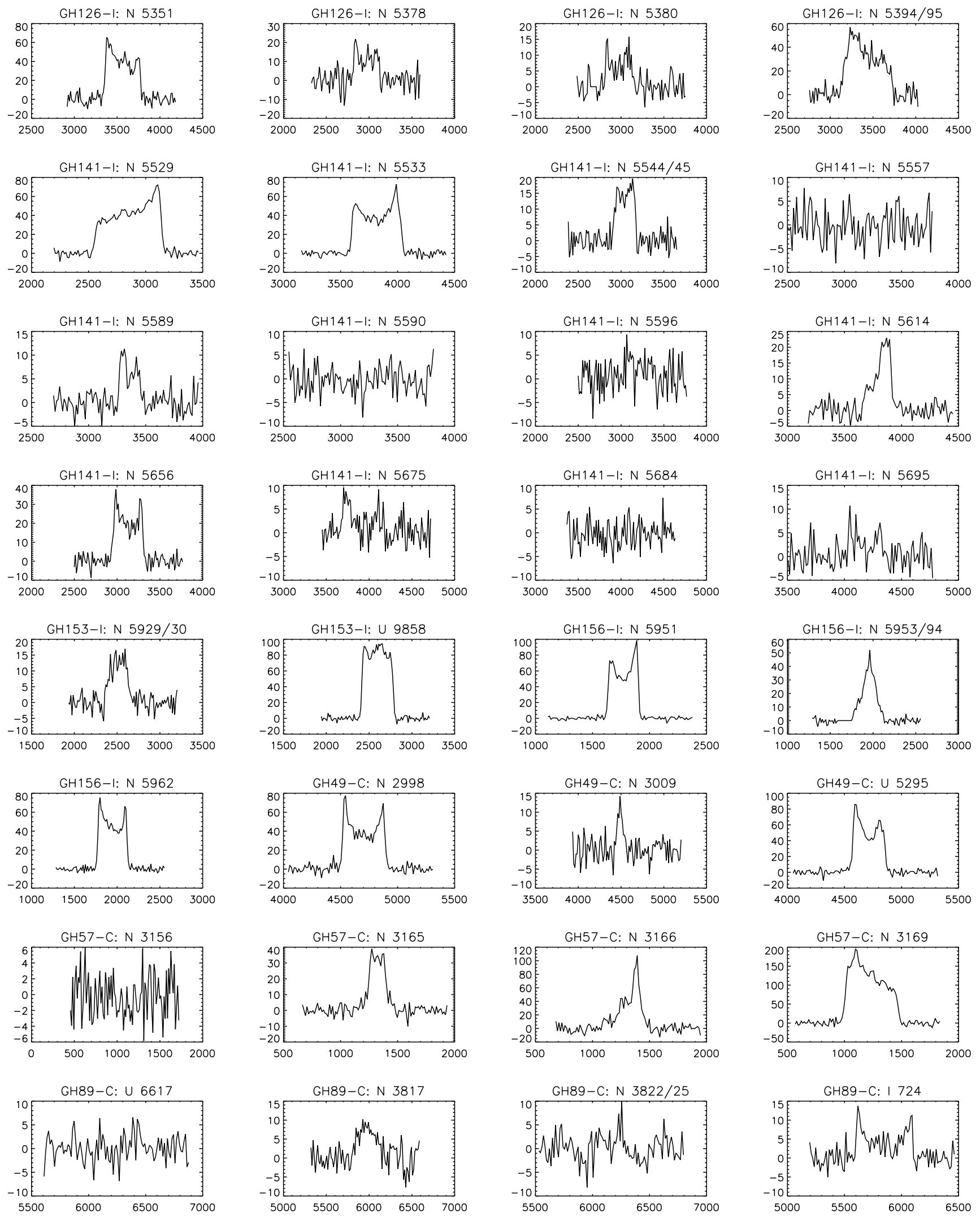

Fig. 1. b) continued.

profile pointed at UGC 6807 is available in the literature In conclusion, no reliable H i profiles of these two objects (Garcia et al. 1994), which will be confused by UGC 6806. are available. 

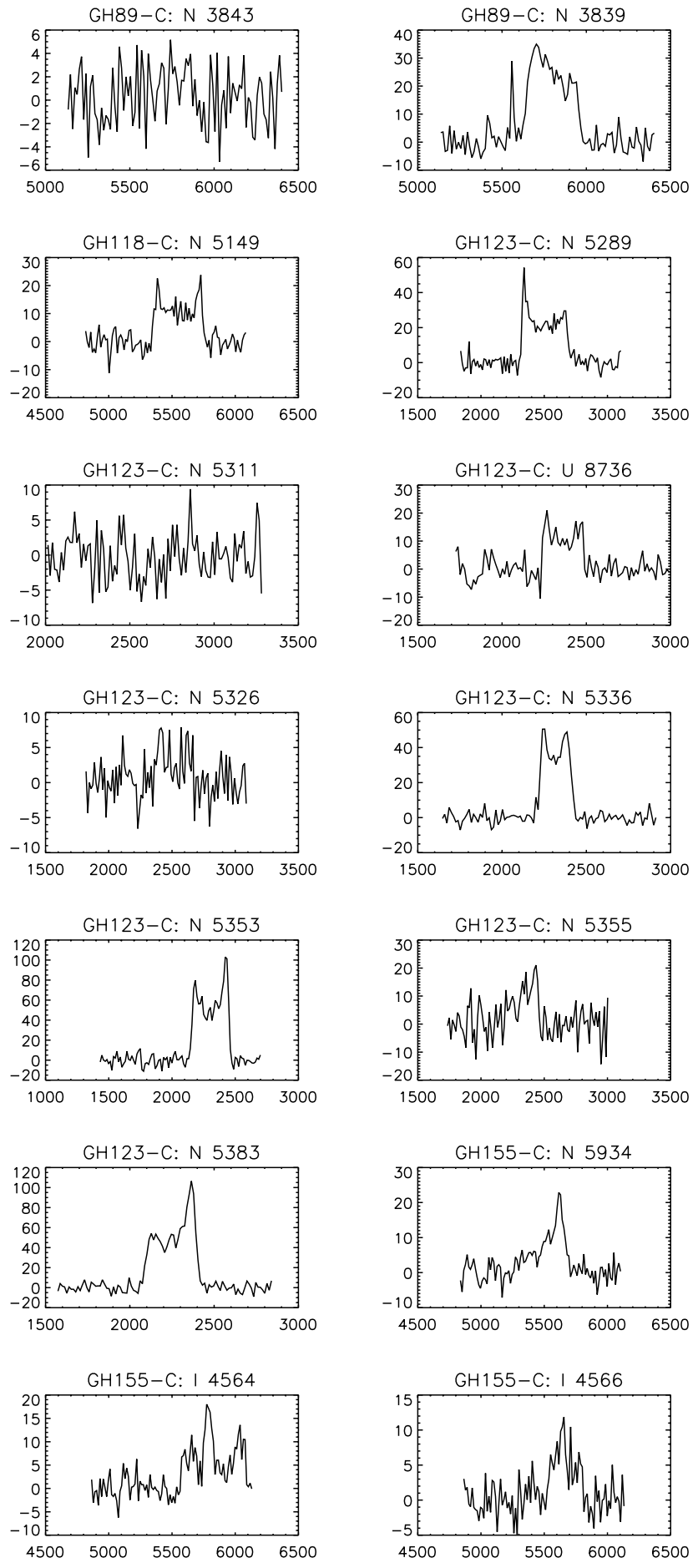

Fig. 1. c) continued.

$N G C$ 3944: E/S0 galaxy, not detected in our survey (estimated $I_{\mathrm{HI}}<3.1$ Jy $\mathrm{km} \mathrm{s}^{-1}, \quad M_{\mathrm{HI}} / L_{B}<$ $\left.0.21 M_{\odot} / L_{\odot, B}\right)$.

$N G C$ 398\%: Our H i velocity is about $50 \mathrm{~km} \mathrm{~s}^{-1}$ lower than the 4 reported Arecibo values, and all profiles have very large widths. The mean values for the Arecibo spectra of NGC 3987 are $V_{\mathrm{HI}}=4500 \mathrm{~km} \mathrm{~s}^{-1}, W_{20}=569 \mathrm{~km} \mathrm{~s}^{-1}$ and $I_{\mathrm{HI}}=7.1 \mathrm{Jy} \mathrm{km} \mathrm{s}^{-1}$. A possible source of confusion is the $B_{\mathrm{T}} 15.8 \mathrm{mag}$ Sbc spiral NGC 3989, at an E-W distance of 1'2 from NGC 3987. NGC 3989 has no published optical
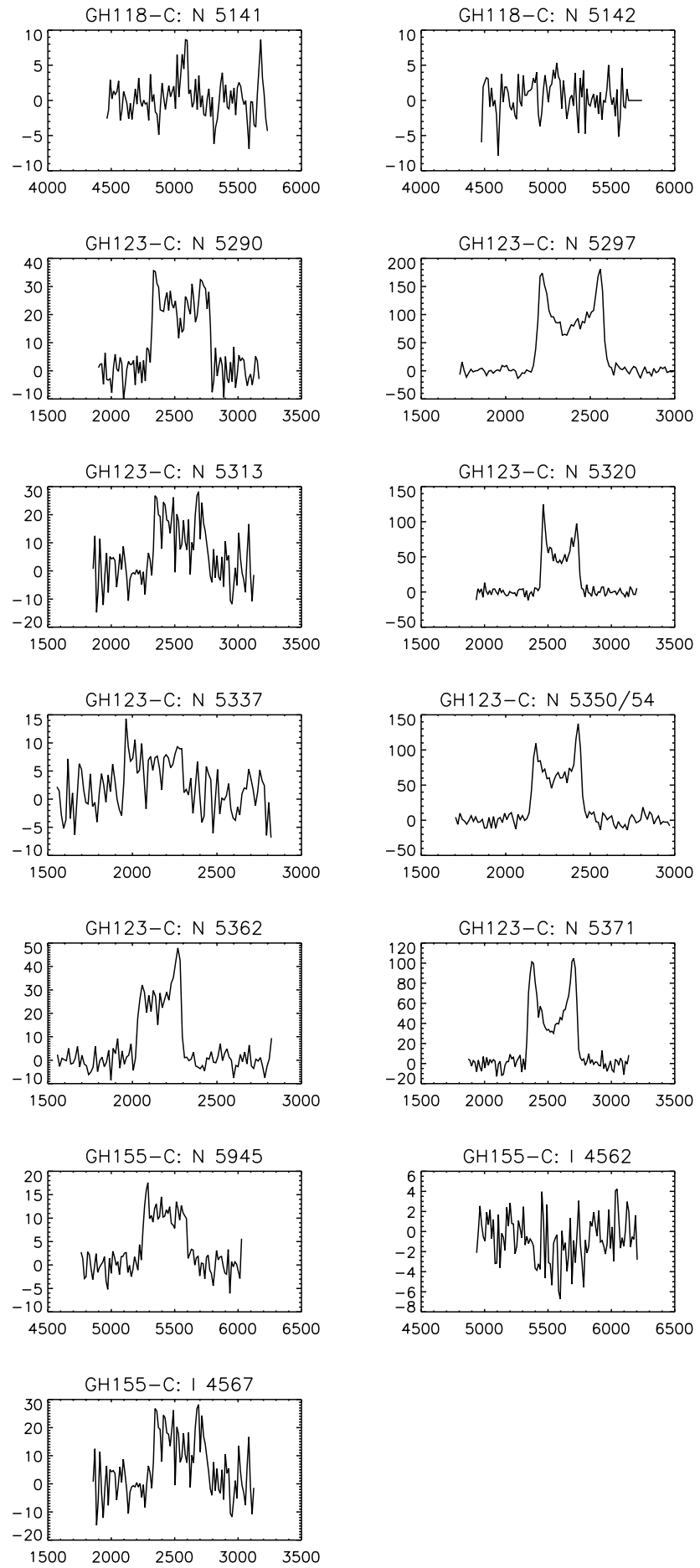

redshift. The Arecibo H I profile parameters for NGC 3989 of Mould et al. (1993) and Scodeggio et al. (1993 - where NGC 3987 is referred to as NGC 3997) are comparable (average $V_{\mathrm{HI}}=4623 \mathrm{~km} \mathrm{~s}^{-1}, W_{20}=519 \mathrm{~km} \mathrm{~s}^{-1}$ and $I_{\mathrm{HI}}=3.2 \mathrm{Jy} \mathrm{km} \mathrm{s}{ }^{-1}$ ), while the Arecibo profile of Williams (1986) has a $90 \mathrm{~km} \mathrm{~s}^{-1}$ higher central velocity $\left(4713 \mathrm{~km} \mathrm{~s}^{-1}\right)$, less flux $\left(2.0 \mathrm{Jy} \mathrm{km} \mathrm{s}^{-1}\right)$ and a considerably smaller width $\left(W_{20}=374 \mathrm{~km} \mathrm{~s}^{-1}\right)$. Confusion between the Arecibo profiles of NGC 3987 and 3989 is in principle possible, as their projected separation is 2.6 ; 

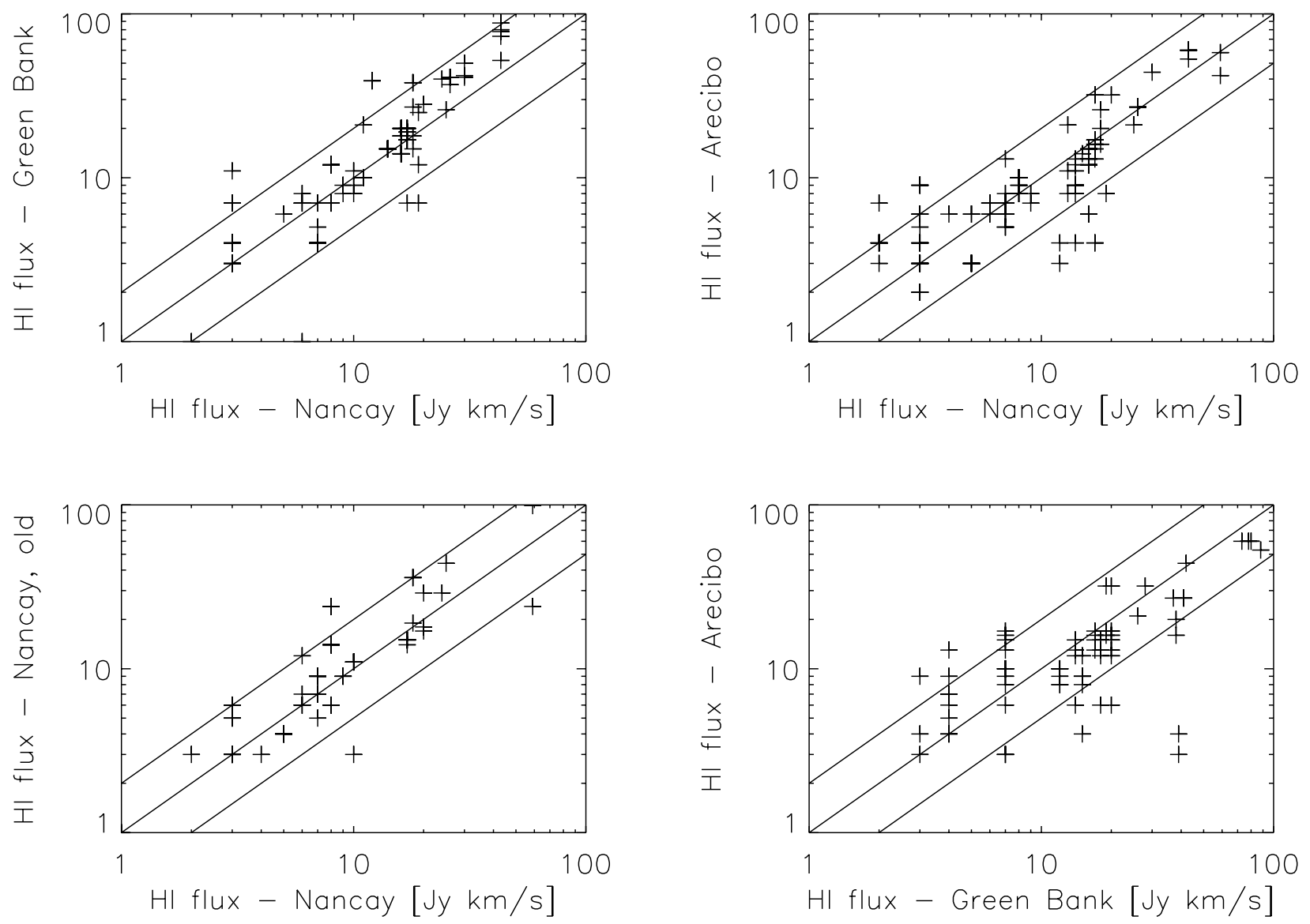

Fig. 2. Comparison of integrated H I line fluxes measured for the present Nançay survey with literature values (see Tables 7 and 8): Green Bank, Arecibo and Nançay, as well as between the literature Green Bank and Arecibo values. Three diagonal lines have been plotted in each panel: the middle line has a slope of unity, the upper line indicates a two times higher flux measured elsewhere than was measured at Nançay, and the lower line a two times lower flux than measured at Nançay. These lines are not fits to the data, they merely serve to guide the eye.

in fact, the spectrum of Scodeggio et al. has a complex structure, indicative of confusion or interaction. On the other hand, the H I emission observed at Arecibo towards NGC 3989 cannot explain the H I observed at Nançay towards NGC 3987 below the low-velocity edge of the Arecibo profile of NGC 3987. In conclusion, the H I distribution appears complex and extended tidal debris may be present in $\mathrm{HI}$.

NGC 3997: our Nançay profile $\left(V_{\mathrm{HI}}=4771 \mathrm{~km} \mathrm{~s}^{-1}\right.$, $\left.W_{20}=241 \mathrm{~km} \mathrm{~s}^{-1}, I_{\mathrm{HI}}=7.6 \mathrm{Jy} \mathrm{km} \mathrm{s}{ }^{-1}\right)$ can in principle be confused by two galaxies: NGC 3993, a $B_{\mathrm{T}} 14.2 \mathrm{mag}$ Sbc spiral with $V_{\text {opt }}=4824 \pm 58 \mathrm{~km} \mathrm{~s}^{-1}$ (LEDA) 2.3 west of NGC 3997 and NGC 4000, a $B_{\mathrm{T}} 15.2 \mathrm{mag}$ Sbc spiral without known $V_{\text {opt }}, 1^{\prime}$ '9 east of NGC 3997. Arecibo observations with a HPBW of 3.3 are available of all three galaxies. These will certainly resolve the confusion between NGC 3997 and NGC 4000 (7.9 separation) and in principle between NGC 3997 and NGC 3993 (2'.8 separation) as well. Measured at Arecibo, the $\mathrm{H}$ I profile parameters of NGC 3997 are $V=4768 \mathrm{~km} \mathrm{~s}^{-1}, W_{20}=$ $289 \mathrm{~km} \mathrm{~s}^{-1}, I_{\mathrm{HI}}=7.9 \mathrm{Jy} \mathrm{km} \mathrm{s}^{-1}$ (Gavazzi 1987; Williams 1986), those of NGC 3993 are $V=4826 \mathrm{~km} \mathrm{~s}^{-1}, W_{20}=$ $382 \mathrm{~km} \mathrm{~s}^{-1}, I_{\mathrm{HI}}=4.3 \mathrm{Jy} \mathrm{km} \mathrm{s}^{-1}$ (Dell'Antonio et al. 1996;
Williams 1986), and those of NGC 4000 are $V=$ $4556 \mathrm{~km} \mathrm{~s}^{-1}, W_{20}=310 \mathrm{~km} \mathrm{~s}^{-1}, I_{\mathrm{HI}}=2.3 \mathrm{Jy} \mathrm{km} \mathrm{s}{ }^{-1}$ (Gavazzi 1987; Williams 1986).

$N G C$ 4005: Sb spiral, not detected clearly in our survey (estimated $I_{\mathrm{HI}}<2.3 \mathrm{Jy} \mathrm{km} \mathrm{s}^{-1}$ ). The 3 published Arecibo detections have almost the same integrated line flux as our estimated $3 \sigma$ upper limit $\left(\sim 1.8 \mathrm{Jy} \mathrm{km} \mathrm{s}^{-1}\right)$.

$N G C 4015 A / B$ : very close pair, with only 0'4 separation between the nuclei; NGC 4015 is seen face-on, while NGC 4015 B is seen edge-on. Their optical redshifts are $4780 \pm 57$ and $4347 \pm 44 \mathrm{~km} \mathrm{~s}^{-1}$, respectively. Both the Arecibo and the Effelsberg spectra (Williams 1986; Huchtmeier et al. 1995) show two distinct peaks, centered on $\sim 4100$ and $\sim 4500 \mathrm{~km} \mathrm{~s}^{-1}$, respectively. In both papers the global line parameters listed refer to the entire emission line profile, resulting in very large $W_{20}$ linewidths of $700-815 \mathrm{~km} \mathrm{~s}^{-1}$. In our spectrum, only the emission centered on $4500 \mathrm{~km} \mathrm{~s}^{-1}$ is present. Huchtmeier et al. considered their profile confused by nearby spirals, but there are no candidates for confusion in the Arecibo or Nançay beams. The origin of the $\mathrm{H}$ I emission at $4100 \mathrm{~km} \mathrm{~s}^{-1}$ is unclear, but could be related to tidal debris. 

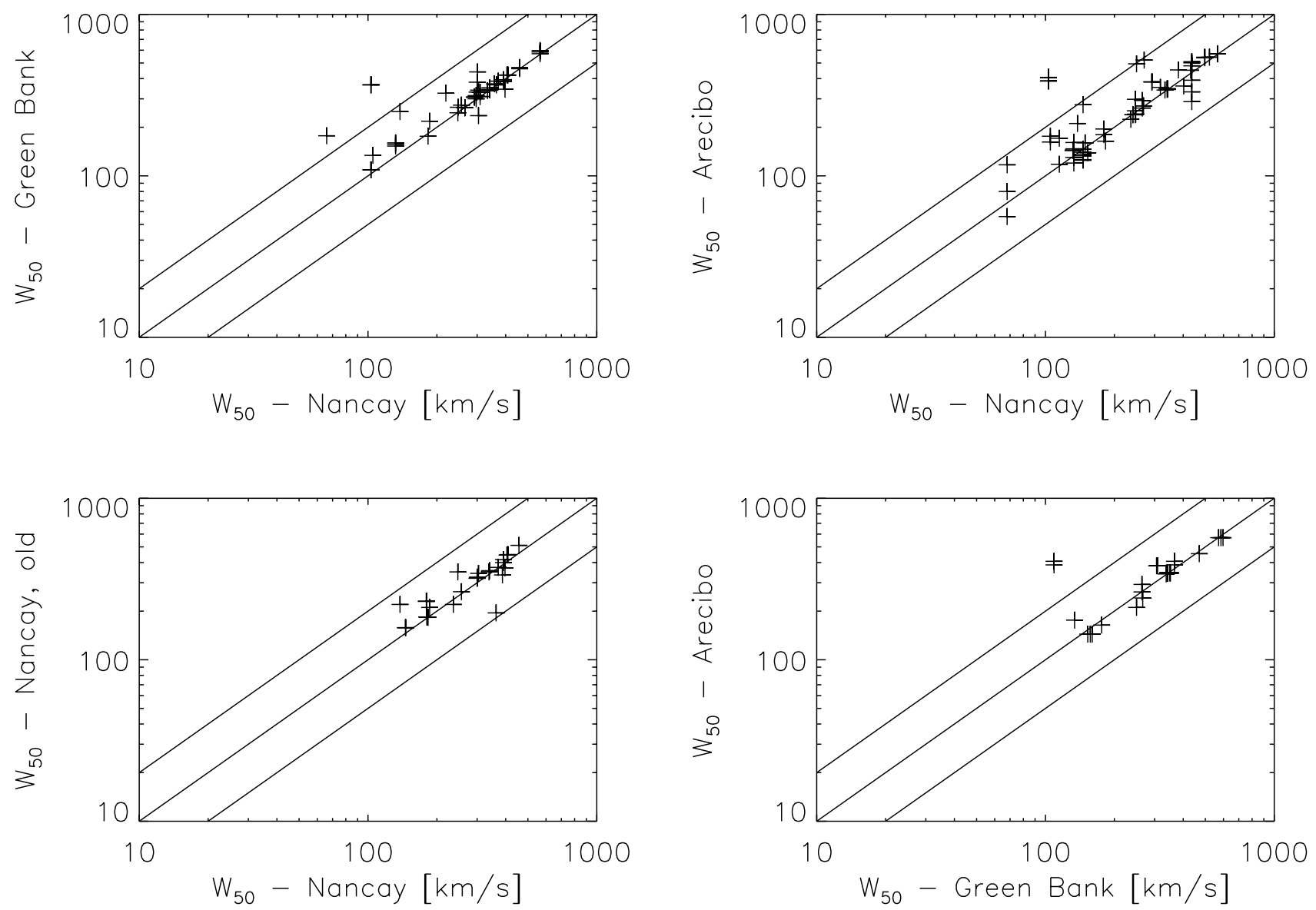

Fig. 3. Comparison of $W_{50} \mathrm{H}$ I line widths measured for the present Nançay survey with literature values (see Tables 7 and 8 ): Green Bank, Arecibo and Nançay, as well as between the literature Green Bank and Arecibo values. Three diagonal lines have been plotted in each panel: the middle line has a slope of unity, the upper line indicates a two times larger $W_{50}$ measured elsewhere than was measured at Nançay, and the lower line a two times smaller $W_{50}$ than measured at Nançay. These lines are not fits to the data, they merely serve to guide the eye.

$N G C$ 4022: S0 galaxy, not detected in our survey (estimated $\left.I_{\mathrm{HI}}<2.6 \mathrm{Jy} \mathrm{km} \mathrm{s}^{-1}, M_{\mathrm{HI}} / L_{B}<0.20 M_{\odot} / L_{\odot, B}\right)$.

\section{Group GH 126:}

$N G C$ 5351: short Westerbork H I synthesis observations (Rhee \& van Albada 1996) show an H I diameter of $4^{\prime} .0$ at a surface density level of $1 M_{\odot} \mathrm{pc}^{-2}, 1.4$ times the optical $D_{25}$ diameter. Though the H I major axis diameter somewhat exceeds the Nançay 3'6 E-W HPBW, all measured integrated line fluxes are in agreement and no significant flux seems to have been missed in our survey.

NGC 5378 and 5380: in principle, our Nançay profiles of these two galaxies are expected to be mutually confused, as they are separated by 11'.4 (about half a HPBW) in the N-S direction. As NGC 5380 has been classified as an elliptical/S0 in various catalogues, we do not expect to detect it in $\mathrm{HI}$; an upper limit of $1.26 \mathrm{Jy} \mathrm{km} \mathrm{s}^{-1}$ was reported for NGC 5380 at Arecibo (Chamaraux et al. 1987), where the 3'6 HPBW should not cause any confusion with NGC 5378. In fact, our detections towards the centres of both objects show strikingly similar profile parameters, indicating they are in fact both detections of the spiral NGC 5378. For their Nançay profile, Theureau et al. (1998), who assumed it to be confused with NGC 5380, listed only a central velocity $\left(2715 \mathrm{~km} \mathrm{~s}^{-1}\right)$, which is about $260 \mathrm{~km} \mathrm{~s}^{-1}$ lower than that of the other two spectra. However, our measurement of the central Hi velocity from the spectrum of Theureau et al. is $2990 \mathrm{~km} \mathrm{~s}^{-1}$, in agreement with the other two spectra, and the linewidths we measured from their spectrum (see Table 6) are in agreement with our Nançay values. The integrated line flux we estimated from the plotted spectrum, $\sim 3.9 \mathrm{Jy} \mathrm{km} \mathrm{s}^{-1}$, is in also agreement with our value. Therefore, only the values measured by us from the Theureau et al. spectrum have been listed in Table 6 . On the other hand, the line flux of NGC 5378 measured at Green Bank by Richter \& Huchtmeier (1991), 11.2 $\mathrm{Jy} \mathrm{km} \mathrm{s}^{-1}$, is much higher than the Nançay values and their profile widths about $40 \%$ larger. This Green Bank profile is not expected to be confused by NGC 5380, given the telescope's HPBW, nor are any other galaxies visible in its vicinity. A broad 

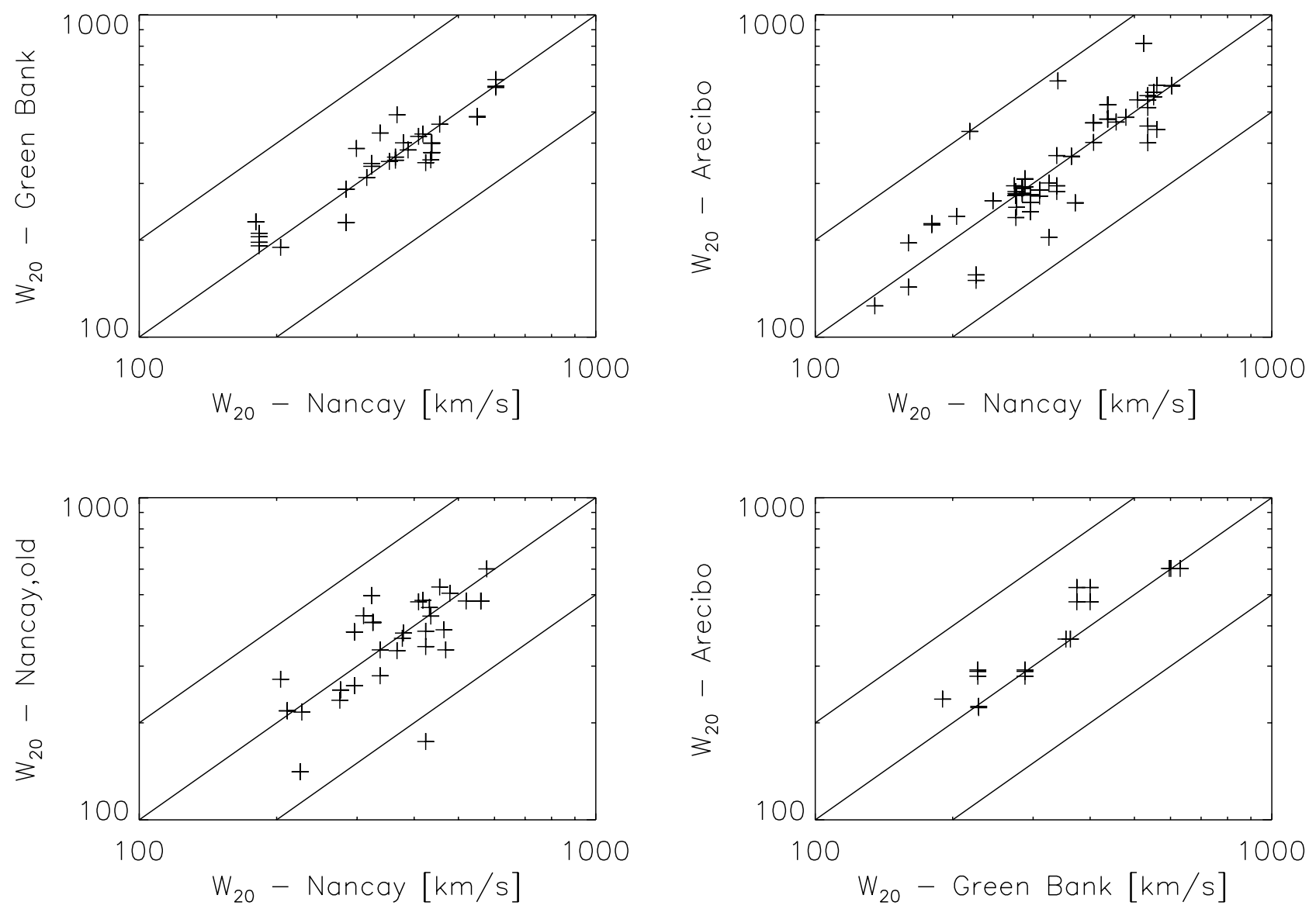

Fig. 4. Comparison of $W_{20} \mathrm{HI}$ line widths measured for the present Nançay survey with literature values (see Tables 7 and 8 ): Green Bank, Arecibo and Nançay, as well as between the literature Green Bank and Arecibo values. Three diagonal lines have been plotted in each panel: the middle line has a slope of unity, the upper line indicates a two times larger $W_{20}$ measured elsewhere than was measured at Nançay, and the lower line a two times smaller $W_{20}$ than measured at Nançay. These lines are not fits to the data, they merely serve to guide the eye.

RFI signal seems the only plausible cause of the striking discrepancy between the Green Bank and Nançay profiles.

\section{Group GH 141:}

$N G C$ 5529: short Westerbork H I synthesis observations (Rhee \& van Albada 1996) show an H I diameter of 6.6 at a surface density level of $1 M_{\odot} \mathrm{pc}^{-2}$, the same as its optical $D_{25}$ diameter. Their position-velocity map indicates that the Nançay beam with its 3'.6 E-W HPBW does not cover all of the $\mathrm{H}$ I emission of this edge-on E-W oriented spiral. In fact, our line flux value of $26.4 \mathrm{Jy} \mathrm{km} \mathrm{s}^{-1}$ is significantly lower than the $36.3 \mathrm{Jy} \mathrm{km} \mathrm{s}^{-1}$ measured at Westerbork, while the latter is in agreement with $\sim 40 \mathrm{Jy} \mathrm{km} \mathrm{s}^{-1}$ measured at Green Bank and Jodrell Bank, whose beams should cover the entire H I disk.

$N G C$ 5533: short Westerbork H I synthesis observations (Broeils \& van Woerden 1994) show that its radial $\mathrm{H}$ I distribution is symmetric and that it extends out to 2.4 times the optical $D_{25}$ diameter of the galaxy (3'2), measured at a surface density level of $1 M_{\odot} \mathrm{pc}^{-2}$. The integrated line flux measured at Westerbork (35.3 $\mathrm{Jy} \mathrm{km} \mathrm{s}^{-1}$ ) is much larger than the Nançay and Green Bank single dish fluxes (19 and $12 \mathrm{Jy} \mathrm{km} \mathrm{s}^{-1}$, respectively), and the
Arecibo flux ( $8 \mathrm{Jy} \mathrm{km} \mathrm{s}^{-1}$ ) is even smaller. The linewidths of the Westerbork and single-dish profiles are comparable, however. The discrepancy between the Westerbork and Green Bank line fluxes is puzzling, as the Green Bank 10.7 HPBW should cover the bulk of the H I emission, seen the 7.7 H I disk major axis measured at Westerbork.

$N G C 5544 / 5$ : Very close interacting pair, the nuclei are separated by only 0'6. Our Nançay values are in agreement with the 7 published Arecibo, Effelsberg and Green Bank profiles, while the Nançay values of Bottinelli et al. (1982) show a considerably higher line flux and linewidths.

$N G C$ 555\%: not detected in our survey (estimated $\left.I_{\mathrm{HI}}<2.6 \mathrm{Jy} \mathrm{km} \mathrm{s}^{-1}\right)$. Classified as an elliptical; the upper limit to its gas content $\left(M_{\mathrm{HI}} / L_{B}<0.02 M_{\odot} / L_{\odot, B}\right)$ is consistent with its classification.

NGC 5589: Though our H I velocity and linewidths are comparable to the Arecibo values listed in the Huchtmeier \& Richter (1989) catalogue, our integrated line flux of $1.5 \mathrm{Jy} \mathrm{km} \mathrm{s} \mathrm{km}^{-1}$ is considerable higher than the uncertain $0.53 \pm 0.36 \mathrm{Jy} \mathrm{km} \mathrm{s}^{-1}$ listed as the Arecibo value. However, this Arecibo reference, referred to as "Richter \& Williams 1989, to be submitted" in the Huchtmeier \& Richter catalogue, has apparently never appeared in print. 
Table 5. Nançay H I line data for the control group sample.

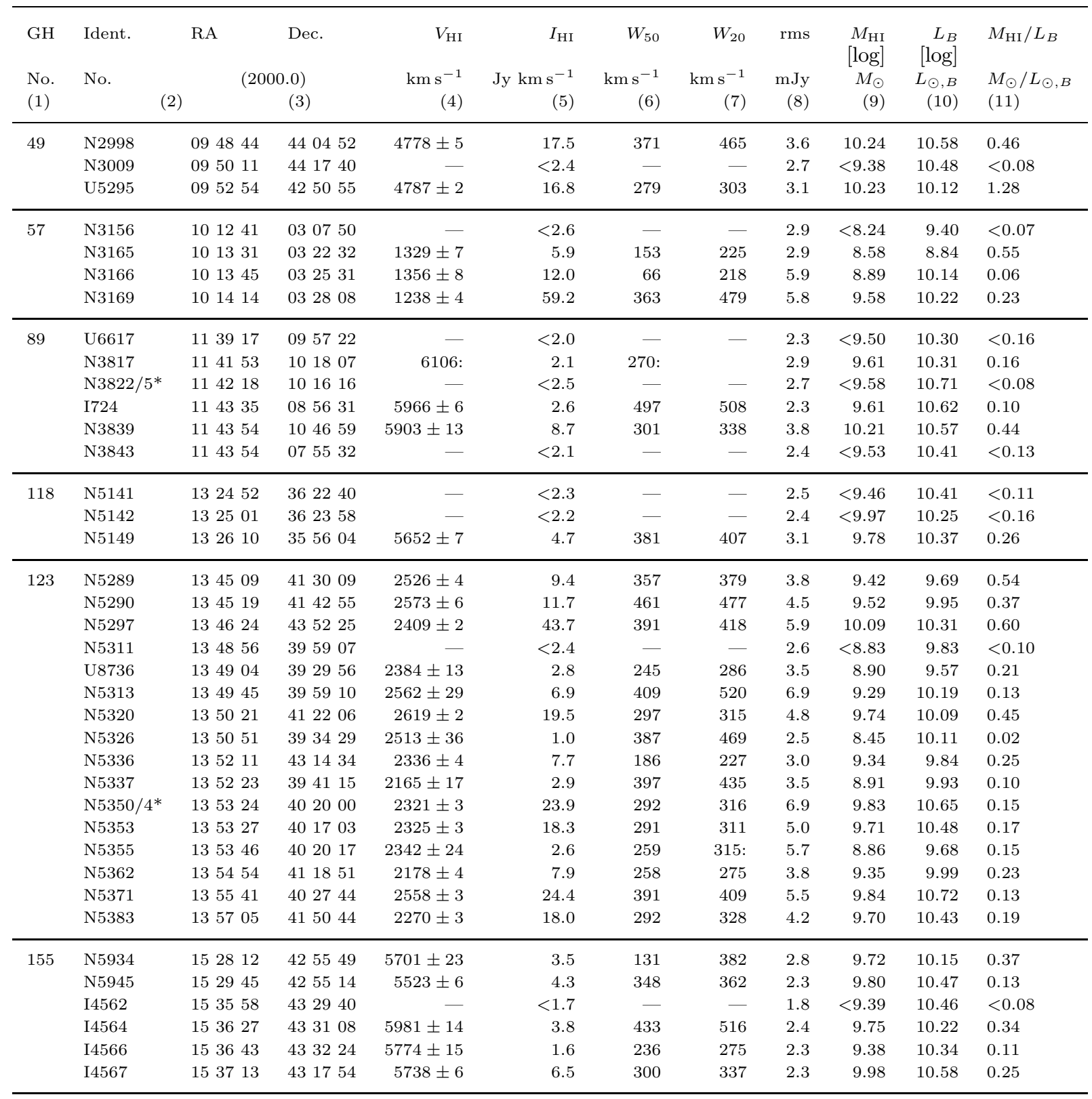

Note: $I_{\mathrm{HI}}$ : upper limits are $3 \sigma$ for $300 \mathrm{~km} \mathrm{~s}^{-1}$ wide, flat-topped profiles; ":" denotes an uncertain value.

No confusion is expected at Nançay from the S0 galaxy NGC 5590 (see below), which lies at an E-W separation of 2.8 from NGC 5589 .

$N G C$ 5590: not detected in our survey (estimated $\left.I_{\mathrm{HI}}<2.8 \mathrm{Jy} \mathrm{km} \mathrm{s}^{-1}\right)$. Classified as a lenticular; the upper limit to its gas content $\left(M_{\mathrm{HI}} / L_{B}<0.10 M_{\odot} / L_{\odot, B}\right)$ is consistent with its classification.

NGC 5614 (=Arp 178): Four of the 5 published H I velocities agree, except for the $\sim 43 \mathrm{~km} \mathrm{~s}^{-1}$ higher Green Bank value by Richter \& Huchtmeier (1991). Our $W_{50}$ linewidth of $138 \mathrm{~km} \mathrm{~s}^{-1}$ is comparable to the Arecibo value listed in Huchtmeier \& Richter (1989) but much smaller than the Green Bank and Nançay literature values of $220-250 \mathrm{~km} \mathrm{~s}^{-1}$, though our $W_{50}$ value of $246 \mathrm{~km} \mathrm{~s}^{-1}$ is comparable to the published values.

$N G C$ 5656: our Nançay values agree with the published Nançay values (Theureau et al. 1998), if we multiply their integrated line flux (6.1 Jy $\mathrm{km} \mathrm{s}^{-1}$ ) by a factor of 1.26 to convert their flux calibration to the one we adopted (see Matthews et al. 2000, and references therein) and with the Arecibo measurement of $7.9 \mathrm{Jy} \mathrm{km} \mathrm{s}^{-1}$ listed in Huchtmeier \& Richter (1989); surprisingly, the galaxy was not detected $\left(I_{\mathrm{HI}} \leq 3.0 \mathrm{Jy} \mathrm{km} \mathrm{s}^{-1}\right)$ at Arecibo by Krumm \& Salpeter (1980).

NGC 5675: this SBb spiral with a LINER spectrum was not detected in our Nançay survey (estimated 
$I_{\mathrm{HI}}<2.2 \mathrm{Jy} \mathrm{km} \mathrm{s}^{-1}$ ), nor at Arecibo (see Table 7). The best upper limit on its $M_{\mathrm{HI}} / L_{B}$ ratio, $0.10 M_{\odot} / L_{\odot, B}$, is quite low for its type.

$N G C$ 5695: this $\mathrm{SBb}$ with a Seyfert2 spectrum was not clearly detected in our Nançay survey (estimated $I_{\mathrm{HI}}<2.4 \mathrm{Jy} \mathrm{km} \mathrm{s}^{-1}$ ), which is consistent with Arecibo detections. The upper limit on its $M_{\mathrm{HI}} / L_{B}$ ratio, $0.10 M_{\odot} / L_{\odot, B}$, is low for its classification.

\section{Group GH 153:}

$N G C 5929 / 30$ (= Arp 90): very close pair (0.5 separation), well within the Nançay beam and clearly detected. NGC 5929 is a $B_{\mathrm{T}} 14.1 \mathrm{mag}$ Sab:pec with a Seyfert2 spectrum and NGC 5930 is a $B_{\mathrm{T}} 13.5 \mathrm{mag} \mathrm{SABbpec}$, and their difference in optical radial velocity is $150 \mathrm{~km} \mathrm{~s}^{-1}$. $\mathrm{H}$ I line absorption was detected towards the nuclear region of NGC 5929 with MERLIN (Cole et al. 1998).

$U G C$ 9858: our integrated line flux (30 Jy $\mathrm{km} \mathrm{s}^{-1}$ ) is lower than the $40-50 \mathrm{Jy} \mathrm{km} \mathrm{s}{ }^{-1}$ measured at Green Bank. This may well be due to missed flux at Nançay, as the E-W $D_{25}$ diameter of the galaxy is about $4^{\prime} 2$, somewhat larger than the E-W HPBW of 3'6. Our center velocity and linewidth are comparable to the published values, however.

\section{Group GH 156:}

A grid of pointings with $5^{\prime}$ spacing in this group was mapped at Arecibo (Zwaan 2000, 2000) as part of a targeted search for H i clouds in galaxy groups. No such clouds were detected at an rms noise level of $\sim 0.75 \mathrm{mJy}$ for a $10 \mathrm{~km} \mathrm{~s}^{-1}$ resolution, implying a $5 \sigma$ upper limit of about $6.5 \times 10^{6} M_{\odot}$ for $\mathrm{H}$ I clouds with an $\mathrm{H}$ I linewidth of $10 \mathrm{~km} \mathrm{~s}^{-1}$ at the distance we adopted for this group, $27.2 \mathrm{Mpc}$.

NGC 5951: short Westerbork H I synthesis observations (Rhee \& van Albada 1996) show an H I diameter of 3 '. at a surface density level of $1 M_{\odot} \mathrm{pc}^{-2}, 1.1$ times the optical $D_{25}$ diameter. Two integrated line flux measurements of the galaxy are considerable higher than the $\sim 18 \mathrm{Jy} \mathrm{km} \mathrm{s}{ }^{-1}$ measured in 5 other studies, for unclear reasons: the $32.5 \mathrm{Jy} \mathrm{km} \mathrm{s}{ }^{-1}$ measured at Arecibo by Freudling (1995) and the $27.6 \mathrm{Jy} \mathrm{km} \mathrm{s}^{-1}$ measured at Jodrell Bank by Staveley-Smith \& Davies (1988).

NGC 5953/4 pair: was mapped in $\mathrm{HI}$ at the VLA (Chengalur et al. 1994). $R$-band CCD imaging shows that the galaxies are joined by a broad stellar bridge, while NGC 5953 shows an extension towards the north-west. At this position angle an H I plume extends from the galaxy. The overall HI distribution is complex, as is the velocity field, particularly that of NGC 5953 and no clear picture can be drawn of the various kinematical components and their relation to the two galaxies. The total measured H I mass of the system is $1.3 \times 10^{9} M_{\odot}$ (equivalent to $F_{\mathrm{HI}}=8.0 \mathrm{Jy} \mathrm{km} \mathrm{s}{ }^{-1}$ ), of which the authors could readily associate $0.2 \times 10^{9}$ and $0.5 \times 10^{9} M_{\odot}$ with NGC 5953 and 5954, respectively. NGC 5953 is an SAa:pec with a LINER/Seyfert2, and NGC 5954 an SAB(rs)cd:pec with a Seyfert2 spectrum.
In our Nançay profile, two adjacent but distinct peaks can be seen, one with $V=1700 \mathrm{~km} \mathrm{~s}^{-1}, I_{\mathrm{HI}}=$ $3.4 \mathrm{Jy} \mathrm{km} \mathrm{s}^{-1}, W_{50}=116 \mathrm{~km} \mathrm{~s}^{-1}$ and $W_{50}=133 \mathrm{~km} \mathrm{~s}^{-1}$, and the other with $V=1966 \mathrm{~km} \mathrm{~s}^{-1}$ and $W_{50}=$ $146 \mathrm{~km} \mathrm{~s}^{-1}$. Nançay and Green Bank profiles centered on the NGC 5953/4 pair will be confused by H I emission from nearby UGC 9902, a $B_{\mathrm{T}} 17 \mathrm{mag}, 0^{\prime} 9$ diameter SBdm? spiral located $3^{\prime}$ due south of the pair. Chengalur et al. (1994) note its VLA detection without giving detailed global profile parameters. At Arecibo, whose small beam should avoid confusion with the NGC 5953/4 pair, Haynes (1981) measured $V=1695 \mathrm{~km} \mathrm{~s}^{-1}, W_{50}=106 \mathrm{~km} \mathrm{~s}^{-1}$ and an integrated H I line flux of $3.4 \mathrm{Jy} \mathrm{km} \mathrm{s}^{-1}$ for UGC 9902 (compared to 8.0 for the NGC 5953/4 pair). We therefore conclude that the $1700 \mathrm{~km} \mathrm{~s}^{-1}$ peak in our spectrum is due to nearby UGC 9902.

$N G C$ 5962: Nançay profiles of this galaxy $(V=$ $1957 \mathrm{~km} \mathrm{~s}^{-1}, I_{\mathrm{HI}}=16 \mathrm{Jy} \mathrm{km} \mathrm{s}^{-1}$ ) will be slightly confused by UGC 9925, a $B_{\mathrm{T}} 15.4 \mathrm{mag}$ Sc spiral 10'1 due south of it. Arecibo H I profiles of UGC 9925 (Lewis et al. 1985; Sulentic \& Arp 1983), which are certainly not confused by NGC 5962 due to the small HPBW, show it has $V=1916 \mathrm{~km} \mathrm{~s}^{-1}, F W H M=182 \mathrm{~km} \mathrm{~s}^{-1}$ and $I_{\mathrm{HI}}=1.9 \mathrm{Jy} \mathrm{km} \mathrm{s}^{-1}$. One Green Bank observation (Magri 1994) has a much smaller line flux (7.0 $\mathrm{Jy} \mathrm{km} \mathrm{s}^{-1}$ ) than the average $16 \mathrm{Jy} \mathrm{km} \mathrm{s}^{-1}$ found in 7 other studies.

\subsubsection{Control group sample}

\section{Group GH 49:}

NGC 2998: short Westerbork observations (Broeils \& van Woerden 1994) show its H I distribution to be symmetric but not very extended compared to its optical dimensions. Full Westerbork H I synthesis mapping (Broeils 1992) show that its $\mathrm{H}$ I disk has a diameter of $3.8\left(1.3 D_{25}\right)$ and a regular velocity field with a classical, flat rotation curve at $V_{\text {rot }}=210 \mathrm{~km} \mathrm{~s}^{-1}$.

Three small spiral companions were detected in $\mathrm{H}$ i at Westerbork: NGC 3002, NGC 3006 and MCG 07-20-057. These are $B_{\mathrm{T}} 15-16$ mag objects at distances of $5^{\prime}$ to $12^{\prime}$ from NGC 2998, of about 0'65 diameter and with integrated $\mathrm{H}$ I fluxes ranging from 1.7 to $5.1 \mathrm{Jy} \mathrm{km} \mathrm{s}^{-1}$ at about the same systemic velocity as NGC 2998 (Broeils 1992). They lie well outside the Nançay HPBW, but can cause some confusion in Green Bank and Jodrell Bank profiles of NGC 2998. Our Nançay profile (estimated $I_{\mathrm{HI}}=17.5 \mathrm{Jy} \mathrm{km} \mathrm{s}^{-1}$ ) agrees well with that of Theureau et al. (1998). The Green Bank (7.0 Jy km s${ }^{-1}$ ) and Jodrell Bank fluxes (31.9 Jy $\mathrm{km} \mathrm{s}^{-1}$ ) are quite different, though central velocities and linewidths are consistent between all available profiles.

$N G C$ 3009: our narrow "detection" seen in Fig. 1b is in fact spurious and due to radio interference, which occurs in the $H$ polarization only. The upper limit listed in Table 6 was derived from the unaffected $V$ polarization data only. This Sc spiral was reported as detected at Green Bank by Haynes et al. (1988), who noted that their 
Table 6. Published H I line data - interacting group sample.

\begin{tabular}{|c|c|c|c|c|c|c|c|c|c|c|c|c|c|c|}
\hline $\mathrm{GH}$ & Ident. & $V_{\mathrm{HI}}$ & $I_{\mathrm{HI}}$ & $W_{50}$ & $W_{20}$ & Tel. & Ref. & Ident. & $V_{\mathrm{HI}}$ & $I_{\mathrm{HI}}$ & $W_{50}$ & $W_{20}$ & Tel. & Ref. \\
\hline $\begin{array}{l}\text { No. } \\
(1)\end{array}$ & $(2)$ & $\begin{array}{l}\mathrm{km} \mathrm{s}^{-1} \\
(3)\end{array}$ & $\begin{array}{r}\mathrm{Jy} \mathrm{km} \mathrm{s}^{-1} \\
(4)\end{array}$ & $\begin{array}{r}\mathrm{km} \mathrm{s}^{-1} \\
(5)\end{array}$ & $\begin{array}{r}\mathrm{km} \mathrm{s}^{-1} \\
(6)\end{array}$ & (7) & (8) & $(2)$ & $\begin{array}{l}\mathrm{km} \mathrm{s}^{-1} \\
(3)\end{array}$ & $\begin{array}{r}\mathrm{Jy}^{\mathrm{km} \mathrm{s}}{ }^{-1} \\
(4)\end{array}$ & $\begin{array}{r}\mathrm{km} \mathrm{s}^{-1} \\
(5)\end{array}$ & $\begin{array}{r}\mathrm{km} \mathrm{s}^{-1} \\
(6)\end{array}$ & (7) & (8) \\
\hline \multirow[t]{6}{*}{45} & N2798* & 1740 & 11.1 & 339 & 348 & G & PS74 & $\mathrm{N} 2798 / 9$ & 1740 & 10.9 & & 380 & G & PS74 \\
\hline & & 1744 & 8.6 & 236 & & G & H82 & N2799* & 1757 & 9.6 & & & G & DS83 \\
\hline & & 1778 & 9.0 & & & G & DS83 & & 1755 & 11.1 & 343 & 385 & $\mathrm{~N}$ & B82 \\
\hline & & 1726 & 11.1: & 274 & 345 & $\mathrm{~N}$ & B80 & & 1865 & 2.0 & 121 & & $\mathrm{~V}$ & N97 \\
\hline & & 1669 & 3.2 & & 175 & $\mathrm{~N}$ & vB85 & N2844 & 1486 & 5.8 & 310 & & G & H82 \\
\hline & & 1747 & 3.0 & 277 & & $\mathrm{~V}$ & N97 & & 1479 & 7.0 & 329 & & G & M94 \\
\hline \multirow[t]{26}{*}{58} & N3162 & 1290 & 21.5 & 164 & 237 & $\mathrm{~A}$ & $\mathrm{BC} 79$ & N3189 & 1373 & 6.2 & & 605 & $\mathrm{~A}$ & WR87 \\
\hline & & 1302 & 26.6: & 176 & 190 & G & DR78 & & 1518 & 6.8 & & & G & DS83 \\
\hline & & 1373 & 6.2 & & 605 & A & WR87 & & 1314 & 4.4 & & 478 & $\mathrm{~N}$ & $\mathrm{BC} 83$ \\
\hline & & 1302 & 28.6 & 178 & 191 & $\mathrm{~J}$ & D80 & & 1320 & 5.1 & & 528 & $\mathrm{~V}$ & W91 \\
\hline & & 1302 & 44.1 & 183 & 273 & $\mathrm{~N}$ & B82 & N3189/90 & 1302 & 4.1 & & 457 & A & Ha81 \\
\hline & N3177 & 1299 & 6.1 & 180 & & A & KS80 & N3193 & & $<0.4$ & & & $\mathrm{~V}$ & W91 \\
\hline & & 1296 & 3.3 & & 204 & $\mathrm{~A}$ & Ha81 & N3213 & 1347 & 1.7 & 160 & & $\mathrm{~A}$ & BG87 \\
\hline & & 1317 & 5.1 & 195 & 301 & $\mathrm{~A}$ & $\mathrm{BC} 79$ & $\mathrm{~N} 3227^{*}$ & & 15.2: & & & $\mathrm{A}$ & DS83 \\
\hline & & 1303 & 6.4: & 230 & 410 & $\mathrm{~N}$ & B82 & & 1169 & $6.8:$ & $387:$ & & $\mathrm{A}$ & $\mathrm{C} 87$ \\
\hline & N3185 & 1218 & 6.1 & 241 & & $\mathrm{~A}$ & $\mathrm{KS} 80$ & & 1146 & 12.8: & & 526: & $\mathrm{A}$ & M82 \\
\hline & & 1234 & 3.4 & & 278 & $\mathrm{~A}$ & Ha81 & & 1146 & & & 526: & $\mathrm{A}$ & MW84 \\
\hline & & 1226 & 3.7 & & 253 & A & WR87 & & 1152 & 13.3: & 407 & 475 & $\mathrm{~A}$ & B79 \\
\hline & & 1237 & 7.3: & 266 & & G & H82 & & 1138 & 28.6: & 364 & 471 & $\mathrm{E}$ & HB75 \\
\hline & & 1239 & 3.5 & & 253 & $\mathrm{~N}$ & BC83 & & 1165 & 18.4: & 109 & 374 & $\mathrm{G}$ & P79 \\
\hline & & 1200 & 3.3 & & 308 & $\mathrm{~V}$ & W91 & & 1148 & 14.1: & & & G & DS83 \\
\hline & N3187 & 1577 & 8.3 & & & $\mathrm{~A}$ & DS83 & & 1106 & 20.2 : & $366:$ & 400: & G & H78 \\
\hline & & 1580 & 10.7 & & 245 & A & WR87 & & 1199 & 18.9: & 258 & 300 & $\mathrm{~J}$ & LD73 \\
\hline & & 1579 & 10.5 & 224 & 276 & $\mathrm{~A}$ & GS85 & & & 20.7 & & & $\mathrm{~V}$ & W95 \\
\hline & & 1579 & 9.6 & & 262 & $\mathrm{~A}$ & Ha81 & N3239 & 754 & 53.0 & 144 & & $\mathrm{~A}$ & He81 \\
\hline & & 1558 & $7.5:$ & & & G & H82 & & 756 & 60.9 & & & $\mathrm{~A}$ & DS83 \\
\hline & & 1581 & 12.6 & & & G & DS83 & & 754 & 88.0 & 160 & 205 & G & FT81 \\
\hline & & 1582 & 10.3 & 219 & 243 & $\mathrm{~J}$ & $\mathrm{~S} 88$ & & 755 & 80.2 : & 153 & 192 & G & S78 \\
\hline & & 1573 & 24.6 : & 220 & 383 & $\mathrm{~N}$ & $\mathrm{~B} 82$ & & 751 & 73.2 : & 157 & 210 & G & DR78 \\
\hline & & 1591 & 14.2 & & 261 & $\mathrm{~N}$ & $\mathrm{BC} 83$ & & 750 & 78.8 & & 197 & G & TC88 \\
\hline & & 1541 & 10.6 & & 277 & $\mathrm{~V}$ & W91 & & 751 & 73.2 & & & G43 & DS83 \\
\hline & N3189 & 1310 & 3.2 & & 441 & A & LS84 & & 751 & & & & G & RD76 \\
\hline \multirow[t]{13}{*}{67} & N3381 & 1627 & 21.0 & 80 & & $\mathrm{~A}$ & KS80 & U6070 & 1849 & 6.2 & 118 & & $\mathrm{~A}$ & BG87 \\
\hline & & 1631 & 8.5 & 56 & 125 & $\mathrm{~A}$ & L87 & N3430 & 1586 & 44.1 & 340 & & $\mathrm{~A}$ & HS82 \\
\hline & & 1630 & 11.5 & 117 & & $\mathrm{~A}$ & M94 & & 1594 & 42.1 & 337 & 351 & G & DR78 \\
\hline & N3395* & 1620 & 20.2 & 176 & 225 & A & L85 & & 1577 & 57.1 & 338 & 370 & $\mathrm{~J}$ & S88 \\
\hline & & 1621 & 16.0 & & 223 & $\mathrm{~A}$ & J87 & & 1583 & & & & G & T78 \\
\hline & & 1631 & 38.4: & 134: & 228 & G & S75 & & 1594 & & & & G & RD76 \\
\hline & & 1621 & & & & G & RD76 & & 1583 & & & & & F71 \\
\hline & & 1605 & & & & G43 & F71 & N3442 & 1729 & 4.2 & 120 & 196 & $\mathrm{~A}$ & $\mathrm{~T} 81$ \\
\hline & & 1625 & & & & $\mathrm{~V}$ & C99 & & 1732 & 3.1 & 147 & & $\mathrm{~A}$ & BG87 \\
\hline & N3396* & 1625 & 26.1 & 162 & & $\mathrm{~A}$ & KS80 & & & 3.1 & 130 & & $\mathrm{~A}$ & BG87 \\
\hline & & 1684 & & & & $\mathrm{~V}$ & C99 & & 1736 & 2.0 & & 143 & $\mathrm{~A}$ & J87 \\
\hline & N3395/6 & & 26.0 & & & $\mathrm{~V}$ & C99 & & 1731 & 9.1 & 161 & & $\mathrm{~A}$ & M94 \\
\hline & N3424 & 1501 & 14.0 & 353 & & A & HS82 & & & & & & & \\
\hline \multirow[t]{6}{*}{86} & N3786* & 2672 & 12.1 & & & $\mathrm{~A}$ & DS83 & N3786* & \multicolumn{2}{|c|}{ confused } & & & $\mathrm{A} / \mathrm{W}$ & OS93 \\
\hline & & 2707 & 9.1 & & 545 & $\mathrm{~A}$ & MW84 & N3788* & 2687 & 9.4 & 501 & 562 & $\mathrm{~A}$ & L85 \\
\hline & & 2725 & 4.9 & 331 & 401 & $\mathrm{~A}$ & L85 & & 2712 & 13.0 & 391 & 516 & $\mathrm{~A}$ & S86 \\
\hline & & 2770 & 8.1: & 288 & 452 & A & S86 & & 2673 & 11.8 & 509 & & $\mathrm{~A}$ & M94 \\
\hline & & 2703 & 9.7 & 480 & & $\mathrm{~A}$ & M94 & & \multirow{2}{*}{\multicolumn{2}{|c|}{ confused }} & & & $\mathrm{A} / \mathrm{W}$ & OS93 \\
\hline & & 2718 & 15.4 & & & $\mathrm{G}$ & DS83 & & & & & & & \\
\hline \multirow[t]{10}{*}{92} & N3902 & 3601 & 8.8 & 239 & 261 & $\mathrm{~A}$ & L85 & N3997 & 4765 & 8.8 & & 295 & $\mathrm{~A}$ & Wi86 \\
\hline & N3920 & 3640 & 6.9 & 184 & 218 & $\mathrm{~N}$ & G94 & & 4771 & 7.0 & & 283 & $\mathrm{~A}$ & GZ87 \\
\hline & U6806 & 3760 & 4.2 & & 265 & A & Wi86 & N4005 & 4458 & 2.0 & & 402 & $\mathrm{~A}$ & Wi86 \\
\hline & I746 & 5027 & 7.4 & 271 & 309 & $\mathrm{~A}$ & L85 & & 4470 & 2.1 & & 463 & $\mathrm{~A}$ & GZ87 \\
\hline & & 5030 & 6.7 & & 310 & $\mathrm{~A}$ & Wi86 & & 4469 & 1.4 & 360 & & $\mathrm{~A}$ & C93 \\
\hline & & 5027 & 7.7 & & 293 & $\mathrm{~A}$ & GZ87 & N4015 & 4368 & 2.8 & 522 & 700 & $\mathrm{E}$ & H95 \\
\hline & N3987 & 4501 & 7.9 & & 557 & $\mathrm{~A}$ & Wi86 & N4015A* & & $<1.3$ & & & $\mathrm{~A}$ & Wi86 \\
\hline & & 4495 & 6.6 & & 575 & A & GZ87 & N4015B* & 4347 & 2.3 & & 815 & $\mathrm{~A}$ & Wi86 \\
\hline & & 4500 & & & 575 & $\mathrm{~A}$ & M93 & N4022 & & $<1.8$ & & & $\mathrm{~A}$ & Wi86 \\
\hline & N3987 & 4502 & 6.7 & 543 & & $\mathrm{~A}$ & C93 & & & & & & & \\
\hline
\end{tabular}

spectrum is probably confused with NGC 3010, which lies well outside the Nançay beam. Their spectrum shows a broad component between $\sim 4470$ and $4900 \mathrm{~km} \mathrm{~s}^{-1}$ at the 8 mJy level and a narrow $\left(\sim 120 \mathrm{~km} \mathrm{~s}^{-1} F W H M\right)$ 17 mJy peak at $\sim 4570 \mathrm{~km} \mathrm{~s}^{-1}$. As the optical velocities of NGC 3009 and 3010 are $4604 \pm 50$ and $4401 \pm 44 \mathrm{~km} \mathrm{~s}^{-1}$, respecively (LEDA), the association of the $4570 \mathrm{~km} \mathrm{~s}^{-1}$ peak with NGC 3009 seems plausible; surprisingly, we did not detect this peak with our $2.9 \mathrm{mJy}$ rms noise level.

$U G C$ 5295: Our integrated line flux (16.8 $\mathrm{Jy} \mathrm{km} \mathrm{s}^{-1}$ ) is comparable to the Green Bank value of $19.6 \mathrm{Jy} \mathrm{km} \mathrm{s}^{-1}$ from Haynes et al. (1988) but considerable higher than 
Table 6. continued.

\begin{tabular}{|c|c|c|c|c|c|c|c|c|c|c|c|c|c|c|}
\hline $\begin{array}{l}\text { GH } \\
\text { No. } \\
(1)\end{array}$ & $\begin{array}{l}\text { Ident. } \\
(2)\end{array}$ & $\begin{array}{l}V_{\mathrm{HI}} \\
\mathrm{km} \mathrm{s}^{-1} \\
(3)\end{array}$ & $\begin{array}{r}I_{\mathrm{HI}} \\
\mathrm{Jy} \mathrm{km} \mathrm{s}^{-1} \\
(4)\end{array}$ & $\begin{array}{r}W_{50} \\
\mathrm{~km} \mathrm{~s}^{-1} \\
(5)\end{array}$ & $\begin{array}{r}W_{20} \\
\mathrm{~km} \mathrm{~s}^{-1} \\
(6)\end{array}$ & $\begin{array}{l}\text { Tel. } \\
(7)\end{array}$ & $\begin{array}{l}\text { Ref. } \\
(8)\end{array}$ & $\begin{array}{l}\text { Ident. } \\
(2)\end{array}$ & $\begin{array}{l}V_{\mathrm{HI}} \\
\mathrm{km} \mathrm{s}^{-1} \\
(3)\end{array}$ & $\begin{array}{r}I_{\mathrm{HI}} \\
\mathrm{Jy} \mathrm{km} \mathrm{s}^{-1} \\
(4)\end{array}$ & $\begin{array}{r}W_{50} \\
\mathrm{~km} \mathrm{~s}^{-1} \\
(5)\end{array}$ & $\begin{array}{r}W_{20} \\
\mathrm{~km} \mathrm{~s}^{-1} \\
(6)\end{array}$ & $\begin{array}{r}\text { Tel. } \\
(7)\end{array}$ & $\begin{array}{l}\text { Ref. } \\
(8)\end{array}$ \\
\hline \multirow[t]{10}{*}{126} & N5341 & 3648 & 6.2 & 246 & & $\mathrm{G}$ & P79 & \multirow[t]{2}{*}{ N5394* } & 3490 & 32.0: & 454 & \multirow{3}{*}{601} & A & KS80 \\
\hline & N5351 & 3630 & 18.4 & 421 & 458 & G & P79 & & 3472 & 18.4 & 511 & & $\mathrm{~N}$ & T98 \\
\hline & & 3377 & 23.1 & 420 & 445 & $\mathrm{~J}$ & S87 & \multirow[t]{8}{*}{ N5395* } & 3490 & 32.0: & 454 & & A & KS 80 \\
\hline & & 3605 & 19.2 & 445 & 528 & $\mathrm{~N}$ & B82 & & 3445 & 16.0 & & \multirow[t]{3}{*}{606} & $\mathrm{~A}$ & SA83 \\
\hline & & 3610 & 20.2 & 407 & 443 & $\mathrm{~W}$ & R96 & & 3544 & 24.5 & & & G & DS83 \\
\hline & & 3611 & & & & G & $\mathrm{T} 78$ & & 3459 & 28.3 & 470 & & G & RH91 \\
\hline & \multirow{2}{*}{ N5378 } & 2947 & 11.2 & 440 & 490 & G & RH91 & & 3498 & 22.9 & 540 & \multirow{4}{*}{$\begin{array}{r}632 \\
681:\end{array}$} & $\mathrm{J}$ & S87 \\
\hline & & 2990 & & 325 & 335 & $\mathrm{~N}$ & T98 & & 3505 & 17.9: & 570: & & $\mathrm{N}$ & B82 \\
\hline & N5380 & & $<1.3$ & & & $\mathrm{~A}$ & $\mathrm{C} 87$ & & 3459 & 29.9 & & & $\mathrm{~N}$ & $\mathrm{C} 77$ \\
\hline & & & $<6.3$ & & & $\mathrm{E}$ & H82 & & & & & & & \\
\hline \multirow[t]{16}{*}{141} & N5529 & 2878 & 41.3 & 597 & 596 & G & FT81 & \multirow[t]{3}{*}{ N5545* } & 3079 & 4.4 & 298 & \multirow[t]{2}{*}{286} & A & F95 \\
\hline & & 2882 & 37.7 & 571 & 606 & $\mathrm{G}$ & S78 & & 3051 & 4.2 & & & G & DS83 \\
\hline & & 2882 & 27.1 & 570 & 603 & $\mathrm{~A}$ & L85 & & 3088 & 6.0 & 350 & 430 & $\mathrm{~N}$ & B82 \\
\hline & & 2888 & 41.0 & 561 & 587 & $\mathrm{~J}$ & S88 & \multirow[t]{2}{*}{ N5589 } & 3394 & 0.53 & 171 & \multirow[t]{2}{*}{186} & A & HR89 \\
\hline & & 2880 & 41.0 & 585 & 630 & G & RH91 & & & $<5.4$ & & & G & RH91 \\
\hline & & 2895 & 36.3 & 557 & 600 & $\mathrm{~W}$ & R96 & \multirow[t]{4}{*}{ N5614 } & 3884 & 2.3 & 126 & \multirow[t]{2}{*}{219} & A & HR89 \\
\hline & \multirow[t]{5}{*}{ N5533 } & 3867 & 8.3 & 451 & 465 & A & Le85 & & 3889 & 3.7 & & & G & DS83 \\
\hline & & 3867 & 8.3 & 451 & 465 & A & L85 & & 3934 & 4.2 & 250 & 285 & G & RH91 \\
\hline & & 3864 & & & & G & $\mathrm{T} 78$ & & 3899 & 5.8: & 220 & & $\mathrm{~N}$ & $\mathrm{~B} 80$ \\
\hline & & 3859 & 12.0 & & & G & RH91 & \multirow[t]{3}{*}{ N5656 } & & $<3.0$ & & & $\mathrm{~A}$ & KS80 \\
\hline & & 3858 & 35.3 & 409 & 439 & $\mathrm{~W}$ & BW94 & & 3156 & 7.9 & 359 & 393 & $\mathrm{~A}$ & HR89 \\
\hline & \multirow[t]{4}{*}{ N5544* } & 3072 & 3.3 & 253 & 286 & $\mathrm{~A}$ & SK78 & & 3150 & 6.1 & 350 & \multirow[t]{3}{*}{366} & $\mathrm{~N}$ & T98 \\
\hline & & 3086 & 4.1 & 239 & 274 & A & L87 & \multirow[t]{2}{*}{ N5675 } & & $<4.5$ & & & A & H83 \\
\hline & & 3078 & 2.2 & & 234 & $\mathrm{E}$ & H97 & & & $<3.1$ & & & A & HR89 \\
\hline & & 3090 & 3.0 & 220 & 245 & $\mathrm{G}$ & RH91 & N5695 & 4225 & 1.6 & & 376 & A & MW84 \\
\hline & N5545* & 3084 & 4.3 & & & $\mathrm{~A}$ & DS83 & & 4255 & 2.7 & 338 & 364 & $\mathrm{~A}$ & HR89 \\
\hline 153 & N5929* & 2561 & 3.1 & 211 & & $\mathrm{E}$ & H82 & U9858 & 2619 & 50.6 & 366 & 381 & G & FT81 \\
\hline & N5930* & 2498 & 4.1 & 325 & 385 & G & RH91 & & 2630 & 41.0 & & & G & DS83 \\
\hline & & 2530 & & & & $\mathrm{~N}$ & T98 & & 2622 & & & & G & $\mathrm{T} 78$ \\
\hline 156 & N5951 & 1776 & 17.9 & & 279 & $\mathrm{~A}$ & Ha81 & N5953* & 1969 & 5.4 & & 283 & A & M88 \\
\hline & & 1782 & 16.0 & 263 & 289 & $\mathrm{~A}$ & GS85 & & 1965 & 7.3 & 136 & & A & C93 \\
\hline & & & 32.5 & 293 & 292 & $\mathrm{~A}$ & F95 & & 1950 & 7.0: & 158: & 235: & $\mathrm{N}$ & B82 \\
\hline & & 1784 & 20.2 & 264 & 227 & G & FT81 & & 1921: & & 280: & & $\mathrm{V}$ & C94 \\
\hline & & 1779 & 19.1 & & 288 & G & TC88 & N5954* & 1935 & 7.2 & & 275 & $\mathrm{~A}$ & $\mathrm{Ha} 81$ \\
\hline & & 1777 & 27.6 & 264 & 277 & $\mathrm{~J}$ & S88 & & 1938 & 5.9 & & & $\mathrm{~A}$ & DS83 \\
\hline & & 1779 & 20.2 & 265 & 282 & W & R96 & & 1960 & 7.4 & 146 & 279 & $\mathrm{~A}$ & L85 \\
\hline & N5962 & 1955 & 17.1: & 346 & & A & $\mathrm{KS} 80$ & & 1955 & 7.8 & 126 & 235 & $\mathrm{~A}$ & GS85 \\
\hline & & 1957 & 15.4 & 345 & & $\mathrm{~A}$ & G87 & & 1971 & 6.9 & 125 & & A & G87 \\
\hline & & 1958 & 13.2 & & 364 & $\mathrm{~A}$ & M88 & & 1964 & 7.2 & 133 & & A & C93 \\
\hline & & 1958 & 16.7 & 343 & & A & C93 & & & 13.1 & 276 & 275 & $\mathrm{~A}$ & F95 \\
\hline & & 1963 & 17.6: & 342 & 354 & $\mathrm{G}$ & $\mathrm{S} 78$ & & 2000 & 4.1: & & & $\mathrm{G}$ & $\mathrm{S} 78$ \\
\hline & & & & & 362 & $\mathrm{G}$ & S78 & & 1969 & 9.1: & & & $\mathrm{N}$ & B82 \\
\hline & & 1948 & 7.0 & 351 & & $\mathrm{G}$ & M94 & & 1903 & & 217 & & $\mathrm{~V}$ & C94 \\
\hline & & 1960 & 15.0 & 350 & 412 & $\mathrm{~N}$ & $\mathrm{~B} 82$ & N5953/4 & & 8.0 & & & $\mathrm{~V}$ & C94 \\
\hline & N5953* & 1964 & 6.5 & 140 & & A & G87 & & & & & & & \\
\hline
\end{tabular}

Note: ":" denotes an uncertain value.

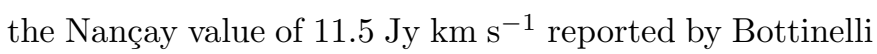
et al. (1982).

\section{Group GH 57:}

NGC 3156/65/66/69: H I in the NGC 3169 group, i.e. the Garcia 192 group (Garcia 1993), was mapped at Arecibo in the NGC $3165 / 66 / 69$ area by Haynes (1981) and extended towards NGC 3156 by Duprie \& Schneider (1996). These observations show complex H I distributions and kinematics: $\mathrm{H}$ I is definitely associated with NGC 3166 and NGC 3166, and detected at and near the position of NGC 3165, but the physical association of this emission with this late-type spiral is unclear. No $\mathrm{HI}$ is detected in the S0 NGC 3156; the rms noise of Duprie \& Schneider, $1.98 \mathrm{mJy}$, gives an upper limit of $1.8 \mathrm{Jy} \mathrm{km} \mathrm{s} \mathrm{s}^{-1}$ to the integrated line flux. The estimated total H I line flux of the group is $128 \mathrm{Jy} \mathrm{km} \mathrm{s}^{-1}$ from the Arecibo mapping of Haynes et al. (1981) and $105 \mathrm{Jy} \mathrm{km} \mathrm{s}{ }^{-1}$ from the Nançay mapping by Balkowski \& Chamaraux (1983). Clearly, the 3 Nançay pointings (centered on NGC 3165, 3166 and 3169) will not cover the extended $\mathrm{H}$ I distribution of the group. The extension of the $\mathrm{HI}$ distribution is also evident from the flux densities of spectra taken in the direction of NGC 3166: $39.1 \mathrm{Jy} \mathrm{km} \mathrm{s}{ }^{-1}$ at Green Bank, 12 at Nançay and 3.5 at Arecibo. Our linewidths for NGC 3166 are much narrower than the 2 literature values, probably due to complex confusion in its vicinity; Haynes (1981) noted a narrow velocity component (presumably associated with the galaxy) superposed on a broad component at and near the galaxy's position. For NGC 3169, compared to our Nançay integrated line flux (59 Jy $\mathrm{km} \mathrm{s}^{-1}$ ), the two published Nançay spectra have either a much larger (99 $\mathrm{Jy} \mathrm{km} \mathrm{s}^{-1}$ ) or a much smaller $\left(24 \mathrm{Jy} \mathrm{km} \mathrm{s}^{-1}\right.$ ) flux, 
and the latter (Bottinelli et al. 1970) has a much smaller $W_{50}$ width, though its $W_{20}$ width is comparable to ours. NGC 3166 is an $\mathrm{SABO}^{-}$a with a LINER spectrum, and NGC 3169 is an SA(s)apec with a LINER spectrum.

\section{Group GH 89:}

$U G C$ 661\%: lenticular galaxy, not detected in our survey (estimated $I_{\mathrm{HI}}<2.0 \mathrm{Jy} \mathrm{km} \mathrm{s}^{-1}$ ). The Arecibo upper limit of $1.5 \mathrm{Jy} \mathrm{km} \mathrm{s}^{-1}$ listed in Table 7 , from Williams (1985), has been corrected to the line width of $300 \mathrm{~km} \mathrm{~s}^{-1}$ used for upper limits throughout the present paper. We assume this to be a $3 \sigma$ upper limit, though this is not mentioned explicitly in Williams' paper.

$N G C$ 3817: our integrated line flux of $2.1 \mathrm{Jy} \mathrm{km} \mathrm{s}^{-1}$ is much higher than the Arecibo value of $1.1 \pm 0.23 \mathrm{Jy} \mathrm{km} \mathrm{s}^{-1}$ reported by Williams (1985), but our detection has a peak flux density of $3.5 \sigma$ only and our line flux is therefore not very accurate.

$N G C$ 3822/5: pair of spirals not clearly detected in our survey (estimated $I_{\mathrm{HI}}<2.4 \mathrm{Jy} \mathrm{km} \mathrm{s}{ }^{-1}$ ). The pair is oriented practically East-West, with a separation of 3.1 , somewhat smaller than the Nançay E-W HPBW and the Arecibo HPBW. Our non-detection is surprising, given the integrated Arecibo line fluxes of 3.6 and $0.8-1.6 \mathrm{Jy} \mathrm{km} \mathrm{s}^{-1}$ reported for NGC 3822 and 3825, respectively, by Eder et al. (1991) and Williams (1985). This may indicated the presence of extended H I emission outside the Nançay beam area. NGC 3822 has a Seyfert2 spectrum.

IC 724: our integrated line flux $\left(2.6 \mathrm{Jy} \mathrm{km} \mathrm{s}^{-1}\right)$ is lower than the $3.5-4.8 \mathrm{Jy} \mathrm{km} \mathrm{s}^{-1}$ measured at Arecibo, and our $W_{50}$ value $\left(497 \mathrm{~km} \mathrm{~s}^{-1}\right)$ is somewhat smaller than the $\sim 540 \mathrm{~km} \mathrm{~s}^{-1}$ measured at Arecibo.

$N G C$ 3843: S0/a galaxy, not detected in our survey (estimated $I_{\mathrm{HI}}<2.1 \mathrm{Jy} \mathrm{km} \mathrm{s}^{-1}$ ). The upper limit to its H I gas content, $M_{\mathrm{HI}} / L_{B}<0.13 M_{\odot} / L_{\odot, B}$, is consistent with its morphological classification.

\section{Group GH 118:}

$N G C 5141$ and $N G C$ 5142: two S0 galaxies, not detected in our survey (estimated $I_{\mathrm{HI}}<2.3$ and $<2.2 \quad \mathrm{Jy} \quad \mathrm{km}^{-1}$, and $M_{\mathrm{HI}} / L_{B}<0.11$ and $<0.16 M_{\odot} / L_{\odot, B}$, respectively).

$N G C$ 5149: Detected at Nançay by Theureau et al. (1998) and in the present survey (at $I_{\mathrm{HI}} 4.7$, $6.6 \mathrm{Jy} \mathrm{km} \mathrm{s}^{-1}$, respectively). Oosterloo \& Shostak (1993) did not detect it at Arecibo, but the Hi line is too weak for a clear detection at their rms level of $6.6 \mathrm{mJy}$; in their short Westerbork observations of the NGC $5149 / 5154$ (= UGC 8444/54) pair the signal of NGC 5149 is confused with that of its companion. No confusion with NGC 5154 is expected for the Nançay spectra, since it has an E-W separation of 3'.8 from NGC 5149.

\section{Group GH 123:}

NGC 5289/90 pair: mapped in $\mathrm{HI}$ at Westerbork (van Moorsel 1983). The H I distribution of NGC 5289 is clearly asymmetric, with an extension towards the NW away from the plane, possibly due to interaction with
NGC 5290, which itself does not seem to be affected. The HI kinematics does not indicate that the interaction was important, as reliable classical flat $\mathrm{H}$ I rotation curves could be derived from the velocity fields: $V_{\text {rot,max }}$ is $\sim 180$ and $225 \mathrm{~km} \mathrm{~s}^{-1}$ for, respectively, NGC 5289 and NGC 5290. The E-W H I diameters of NGC 5289 and 5290 measured at Westerbork are 3.9 and 37, respectively. The 3.6 Nançay E-W HPBW should therefore cover the entire H I emission of each object, as shown by the agreement between our profile parameters and the literature values.

$N G C$ 5311: S0/a galaxy, at $V_{\mathrm{opt}}=2698 \pm 40 \mathrm{~km} \mathrm{~s}^{-1}$, not detected in our survey (estimated $I_{\mathrm{HI}}<$ $2.4 \mathrm{Jy} \mathrm{km} \mathrm{s}^{-1}$ ). Detected at Green Bank (Richter \& Huchtmeier 1991), with a considerably higher integrated line flux, $5.1 \mathrm{Jy} \mathrm{km} \mathrm{s}^{-1}$, at $V=2645 \mathrm{~km} \mathrm{~s}^{-1}$, with $W_{50}=453 \mathrm{~km} \mathrm{~s}^{-1}$. The Green Bank profile could in principle be confused with NGC 5313, a $B_{\mathrm{T}}$ $12.8 \mathrm{mag}$, Sbc type spiral 9.3 due East of NGC 5311, with $V_{\mathrm{opt}}=2597 \pm 44 \mathrm{~km} \mathrm{~s}^{-1}$. Three H I detections of NGC 5313 are available: one from Green Bank (Peterson 1979) and two from Nançay (Bottinelli et al. 1982; the present survey); in principle, the Nançay detections should not be confused by emission from NGC 5311. The H I profile parameters of NGC 5313 are $V=2551 \mathrm{~km} \mathrm{~s}^{-1}$, $I_{\mathrm{HI}}=9.5 \mathrm{Jy} \mathrm{km} \mathrm{s}{ }^{-1}$ and $W_{50}=421 \mathrm{~km} \mathrm{~s}^{-1}$. Because the separation between the two galaxies is comparable to the size of the Green Bank beam, the "detection" of NGC 5311 could be due to the higher velocities in the NGC 5313 profile.

NGC 5320: of the 4 the available Green Bank, Jodrell Bank and Nançay profiles, the Green Bank profile of Magri (1994) is discrepant, having 3.5 times smaller integrated line flux $\left(7 \mathrm{Jy} \mathrm{km} \mathrm{s}{ }^{-1}\right)$, though its central velocity and linewidths are comparable to those of the others.

NGC 5326/5337: short Westerbork H I synthesis observations were made of this pair (Oosterloo \& Shostak 1993), but the galaxies were not detected at an rms noise level of $3.0 \mathrm{mJy} /$ beam (resolution $25^{\prime \prime}$ ).

NGC 5336 our integrated line flux $\left(7.7 \mathrm{Jy} \mathrm{km} \mathrm{s}{ }^{-1}\right)$ is higher than the $5.4 \mathrm{Jy} \mathrm{km} \mathrm{s}{ }^{-1}$ measured at Nançay by Theureau et al. (1998). This general tendency, due to differences in calibration procedures, has already been discussed in Matthews et al. (2000), and references therein.

NGC 5350/3/4/5 area: 5 galaxies constitute Hickson Compact Group 68: NGC 5350, 5353, 5354, 5355 and 5358 (Hickson 1982). NGC 5350/3/4: three closeby galaxies are located near the centre of the Nançay beam in the pointings towards NGC 5350/4 and NGC 5353: the SBb spiral NGC $5350\left(V_{\mathrm{opt}}=2321 \pm 61 \mathrm{~km} \mathrm{~s}^{-1}\right)$ and the early-type $(\mathrm{E} / \mathrm{S} 0)$ systems NGC $5353\left(V_{\mathrm{opt}}=2166 \pm 103 \mathrm{~km} \mathrm{~s}^{-1}\right)$ and NGC $5354\left(V_{\mathrm{opt}}=2681 \pm 244 \mathrm{~km} \mathrm{~s}^{-1}\right)$. In principle, Arecibo or radio synthesis observations could separate the $\mathrm{H}$ I emission from the galaxies, but no Arecibo data are available (see Table 7), and no mention was made of the detection of $\mathrm{HI}$ in either NGC 5353 or NGC 5354 in the short Westerbork observations of NGC 5350 (Rhee \& van Albada 1996). As we do not expect the two earlytypes to be detectable in HI, and as our two Nançay 
Table 7. Published H i line data - control group sample.

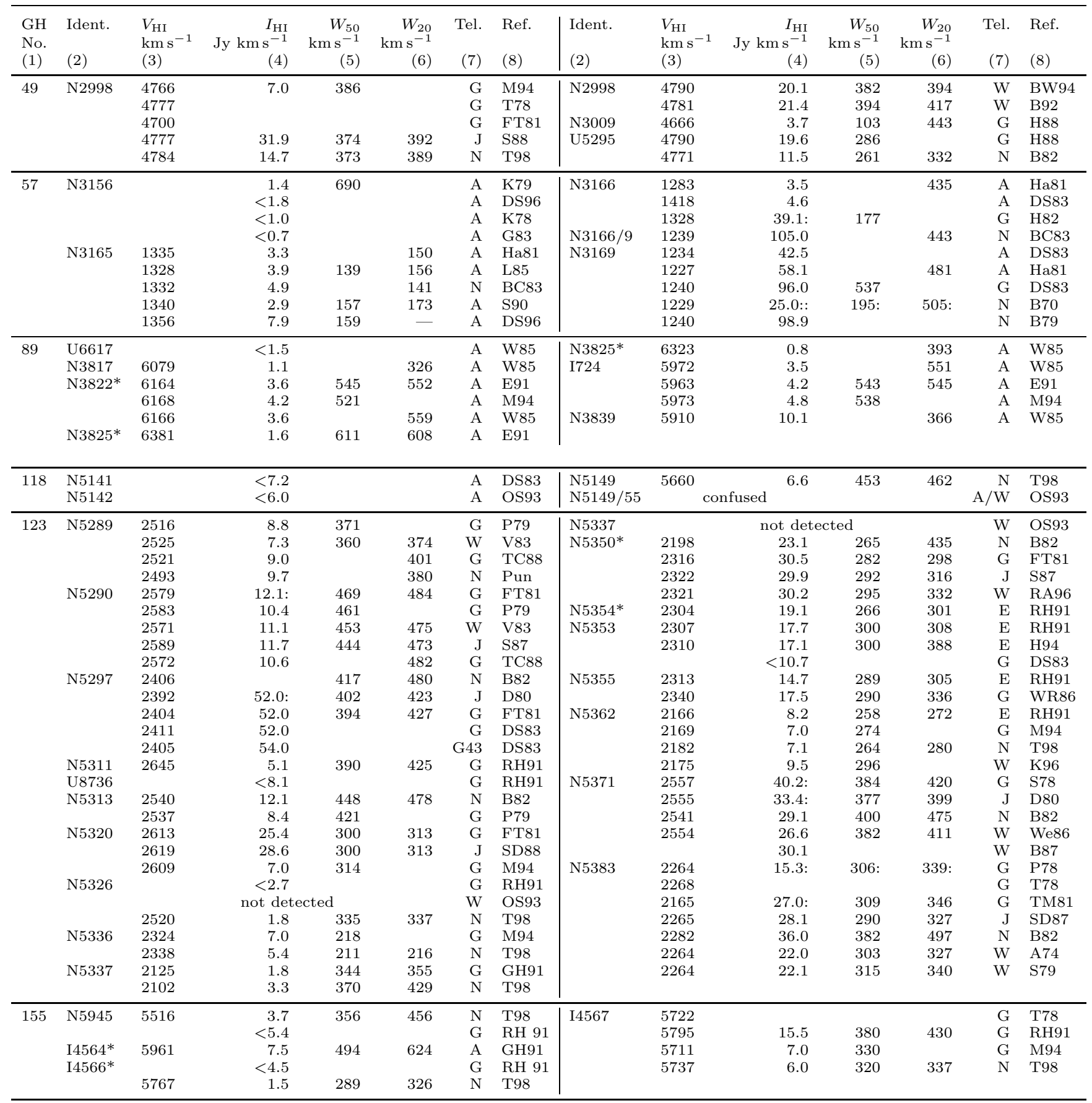

Note: ":" denotes an uncertain value.

profiles have very similar profile parameters, we will assume that all gas detected in our Nançay profiles actually resides in NGC 5350. The Rhee \& van Albada Westerbork data show an $\mathrm{HI}$ diameter of $4^{\prime} 4$ at a surface density level of $1 M_{\odot} \mathrm{pc}^{-2}, 1.1$ times the optical $D_{25}$ diameter. NGC 5355: we detected H I in the Nançay spectrum taken towards NGC 5355, an early-type galaxy (classified as S0 or E). Gas-rich S0 galaxies, though rare, do exist (e.g., Wardle \& Knapp 1985), and often with large H I distributions (van Driel \& van Woerden 1991), but our NGC 5355
H I profile closely resembles the high-velocity half of our strong detection of the large spiral NGC 5350, located 4.5 W of NGC 5355, though with a six times reduced flux density. We therefore consider this "detection" to be of nearby NGC 5350. In principle, our NGC 5355 Nançay spectrum might be confused with $B_{\mathrm{T}} 14.6 \mathrm{mag}$, S0/a NGC 5358, located 3'.0 E of it, but the only H I "detection" of this object (Richter \& Huchtmeier 1991) seems spurious, as its central velocity of $1547 \mathrm{~km} \mathrm{~s}^{-1}$ is completely discrepant with the optical velocities of all nearby galaxies, 
including that of NGC 5358 itself $\left(2432 \pm 60 \mathrm{~km} \mathrm{~s}^{-1}\right.$, based on 2 published measurements).

$N G C$ 5362: short Westerbork H I synthesis observations (Kamphuis et al. 1996) were obtained of this object to determine global H I profile parameters (see Table 7); no further morphological or kinematical H I data are given.

$N G C$ 5371: this $\mathrm{SAB}(\mathrm{rs})$ bc with a LINER spectrum, was mapped in H I at Westerbork (Wevers et al. 1986). Its optical luminosity profile shows a sharp edge at a radius of about $100^{\prime \prime}$, beyond which the surface brightness drops rapidly and the disk becomes redder. Its $\mathrm{H}$ I distribution is ring-shaped with a maximum surface density of $80^{\prime \prime}$ radius, and having an outer edge coinciding with the optical edge. Its velocity field shows signs of a mild warp, and a flat rotation curve was derived from it with a $V_{\text {rot,max }}$ of $300 \mathrm{~km} \mathrm{~s}^{-1}$.

NGC 5383: this barred $\mathrm{SBb}$ system was mapped in $\mathrm{H}$ I at Westerbork (Sancisi et al. 1979). The atomic hydrogen is largely concentrated in the optically bright central parts, and deficient in the region of the bar. Its kinematics are regular, with deviations of order $50-100 \mathrm{~km} \mathrm{~s}^{-1}$ near the bar (similar to those found in other barred galaxies). A classical flat rotation curve was derived from the velocity field with $V_{\text {rot,max }}=210 \mathrm{~km} \mathrm{~s}^{-1}$.

At Westerbork, H I emission was also detected from the SBdm companion UGC 8877, located $3^{\prime}$ South of NGC 5383. The published single-dish $\mathrm{HI}$ data for NGC 5383, as well as our data, will be only slightly confused by its presence, as its integrated HI flux is $1.7 \mathrm{Jy} \mathrm{km} \mathrm{s}^{-1}$, i.e. $8 \%$ of the integrated $\mathrm{HI}$ flux of NGC 5383 as measured at Westerbork. Its central velocity, $2370 \mathrm{~km} \mathrm{~s}^{-1}$, is $120 \mathrm{~km} \mathrm{~s}^{-1}$ higher than that of NGC 5383 . The integrated H I line flux we measured for NGC 5383 (18.0 Jy $\mathrm{km} \mathrm{s}^{-1}$ ) is comparable to the Westerbork value of $22.0 \mathrm{Jy} \mathrm{km} \mathrm{s}^{-1}$, though it is twice as small as the previously published Nançay value (Bottinelli et al. 1982).

\section{Group GH 155:}

$N G C$ 5934: our Nançay profile, with $V_{\mathrm{HI}}=$ $5731 \mathrm{~km} \mathrm{~s}^{-1}$ and $W_{50}=131 \mathrm{~km} \mathrm{~s}^{-1}$, centered on this $B_{\mathrm{T}}$ $14.6 \mathrm{mag}$ Sb spiral which has a $V_{\mathrm{opt}}=5603 \pm 33 \mathrm{~km} \mathrm{~s}^{-1}$, could in principle be confused with that of two galaxies of about similar magnitude and optical diameter: NGC 5935, a nearby (1'.1 separation) $B_{\mathrm{T}} 15.1 \mathrm{mag}$ Sb spiral having an optical redshift of $5373 \pm 60 \mathrm{~km} \mathrm{~s}^{-1}$ (Falco et al. 1999), and PGC 55173 (CGCG 222-012), a $B_{\mathrm{T}} 15.5 \mathrm{mag}$ Sb spiral at 4.7 due north having no published optical redshift. No published $\mathrm{H}$ I data are available for either of these objects. The close NGC 5934/5 pair actually shows signs of interaction on the blue POSS image, as the outer regions of NGC 5934 are turned towards NGC 5935, which appears to have a faint, stubby tail pointing away from NGC 5934. VLA continuum observations at 2 and $6 \mathrm{~cm}$ wavelength (Batuski et al. 1992) detected NGC 5934 only; these authors classified it as an elliptical(?). No optical emission lines were found in NGC 5934 (Sanduleak \& Pesch 1987).

$I C$ 4567: of the 5 available Nançay and Green Bank data, only the Green Bank profile of Richter \& Huchtmeier (1991) is discrepant, with a $\sim 70 \mathrm{~km} \mathrm{~s}^{-1}$ higher central velocity, considerable larger widths and 2.4 times larger integrated line flux (15.5 $\mathrm{Jy} \mathrm{km} \mathrm{s}^{-1}$ ); radio interference may well be the cause of this. There are no galaxies in the vicinity to cause confusion in the $\mathrm{H}$ I observations.

$I C$ 4562: elliptical galaxy, not detected in our survey (estimated $I_{\mathrm{HI}}<1.08 \mathrm{Jy} \mathrm{km} \mathrm{s}^{-1}$ and $M_{\mathrm{HI}} / L_{B}<$ $\left.0.08 M_{\odot} / L_{\odot, B}\right)$.

$I C 4564 / 66$ : first, a single spectrum was obtained pointed towards the mean position of the galaxies' centers. Given the relatively large E-W separation of the galaxies in $\alpha(2$ ' 8$)$ compared to the E-W HPBW of 3'6, spectra were also obtained individually for the center position of each object. These data were used for Table 6 .

$I C 456 \%$ : of the 5 available Nançay and Green Bank data, only the Green Bank profile of Richter \& Huchtmeier (1991) is discrepant, with a $\sim 70 \mathrm{~km} \mathrm{~s}^{-1}$ higher central velocity, considerable larger widths and 2.4 times larger integrated line flux (15.5 $\mathrm{Jy} \mathrm{km} \mathrm{s}^{-1}$ ). Radio interference may well be the cause of this. There are no galaxies in the vicinity to cause confusion in the $\mathrm{H}$ I observations.

\subsection{Integrated $\mathrm{H}$ I line fluxes}

Aside from calibration errors, uncertainties in the integrated line flux measurements include contributions from systematic measurement errors, baseline subtraction uncertainties, the effects of a finite telescope beam, as well as external errors and various degrees of confusion with emission from nearby galaxies. In Fig. 2 we compare our new raw, integrated Nançay H I fluxes with those from other sources (see Tables 6 and 7). Panels $a, b$ and $c$ show our new fluxes versus, respectively, previous Arecibo, Green Bank 90-m and Nançay measurements, while (for comparison) panel $d$ shows a comparison between Green Bank and Arecibo measurements. This provides us with an internal comparison, using the same telescope, as well as an external comparison with other instruments.

All cases where we measured a Nançay flux more than twice as large or more than twice as small as a flux reported in the literature are discussed in Sect. 4.1. These discrepancies are usually related to the presence of extended H I structures in the groups. In a few cases, a single Green Bank measurement differs significantly from other measurements made with the same telescope, which are consistent with our Nançay value. In one case only there is no clear explanation for the discrepancies between line fluxes: NGC 5533, where the Green Bank and Nançay values are much lower than the Westerbork measurement, though the Green Bank beam should have covered the H I disk.

The cases in which large differences were found between line fluxes measured by us and those reported in the literature are: (1) the Nançay-Green Bank comparison shows 5 galaxies or close galaxy pairs with an integrated Green Bank line flux at least twice as large as our Nançay value (in order of increasing Green Bank flux: NGC 3185, 5378, 3166, 3395 and 3239), and 3 galaxies with a Green 
Table 8. References and telescope codes to Tables 6 and 7 .

\begin{tabular}{|c|c|c|c|c|c|}
\hline A74 & Allen et al. (1974) & B79 & Balkowski (1979) & $\mathrm{BC} 83$ & Balkowski \& Chamaraux (1983) \\
\hline $\mathrm{B} 87$ & Begeman (1987) & BG87 & Bicay \& Giovanelli (1987) & $\mathrm{BC} 79$ & Biermann et al. (1979) \\
\hline B70 & Bottinelli et al. (1970) & B80 & Bottinelli et al. (1980) & B82 & Bottinelli et al. (1982) \\
\hline B92 & Broeils (1992) & BW94 & Broeils \& van Woerden (1994) & $\mathrm{C} 77$ & Chamaraux (1977) \\
\hline $\mathrm{C} 87$ & Chamaraux et al. (1987) & C93 & Chengalur et al. (1993) & C94 & Chengalur et al. (1994) \\
\hline C99 & Clemens et al. (1999) & D80 & Davies (1980) & DS83 & Davis \& Seaquist (1983) \\
\hline DR78 & Dickel \& Rood (1978) & DS96 & Duprie \& Schneider (1996) & E91 & Eder et al. (1991) \\
\hline FT81 & Fisher \& Tully (1981) & F71 & Ford et al. (1971) & F95 & Freudling (1995) \\
\hline G87 & Garwood et al. (1987) & G94 & Garcia et al. (1994) & GZ87 & Gavazzi (1987) \\
\hline G83 & Giovanardi et al. (1983) & GS85 & Giovanardi \& Salpeter (1985) & Ha81 & Haynes (1981) \\
\hline $\mathrm{H} 88$ & Haynes et al. (1988) & $\mathrm{H} 78$ & Heckman et al. (1978) & H83 & Heckman et al. (1983) \\
\hline H94 & Huchtmeier (1994) & H97 & Huchtmeier (1997) & HR89 & see Huchtmeier \& Richter (1989) \\
\hline He81 & Helou et al. (1981) & HS 82 & Helou et al. (1982) & H82 & Huchtmeier (1982) \\
\hline H95 & Huchtmeier et al. (1995) & HB75 & Huchtmeier \& Bohnenstengel (1975) & J87 & Jackson et al. (1987) \\
\hline K78 & Knapp et al. (1978) & K79 & Knapp et al. (1979) & K96 & Kamphuis et al. (1996) \\
\hline KS 80 & Krumm \& Salpeter (1980) & LS84 & Lake \& Schommer (1984) & Le85 & Lewis (1985) \\
\hline L85 & Lewis et al. (1985) & L87 & Lewis (1987) & LD73 & Lewis \& Davies (1973) \\
\hline M94 & Magri (1994) & M82 & Mirabel (1982) & MW84 & Mirabel \& Wilson (1984) \\
\hline M88 & Mirabel \& Sanders (1988) & M93 & Mould et al. (1993) & N97 & Nordgren et al. (1997) \\
\hline OS93 & Oosterloo \& Shostak (1993) & PS74 & Peterson \& Shostak (1974) & P78 & Peterson et al. (1978) \\
\hline P79 & Peterson (1979) & Pun & Paturel (1998) & R96 & Rhee \& van Albada (1996) \\
\hline RH91 & Richter \& Huchtmeier (1991) & RD76 & Rood \& Dickel (1976) & S79 & Sancisi et al. (1979) \\
\hline S86 & Schneider et al. (1986a) & S90 & Schneider et al. (1990) & S75 & Shostak $(1975)$ \\
\hline S78 & Shostak (1978) & SK78 & Silverglate \& Krumm (1978) & S87 & Staveley-Smith \& Davies (1987) \\
\hline S 88 & Staveley-Smith \& Davies (1988) & SA83 & Sulentic \& Arp (1983) & $\mathrm{T} 78$ & Thonnard et al. (1978) \\
\hline T81 & Thuan \& Martin (1981) & T98 & Theureau et al. (1998) & TC88 & Tifft \& Cocke (1988) \\
\hline vB85 & van der Burg (1985) & V83 & van Moorsel (1983) & W85 & Williams (1985) \\
\hline We86 & Wevers et al. (1986) & Wi86 & Williams (1986) & WR87 & Williams \& Rood (1987) \\
\hline W91 & Williams et al. (1991) & & & & \\
\hline A & Arecibo 305 m & $\mathrm{E}$ & Effelsberg $100 \mathrm{~m}$ & G & Green Bank 91 m \\
\hline G43 & Green Bank 43 m & $\mathrm{J}$ & Joddrell Bank $64 \mathrm{~m}$ & $\mathrm{~N}$ & Nançay $94 \mathrm{~m}$ equiv \\
\hline V & VLA & $\mathrm{W}$ & Westerbork & & \\
\hline
\end{tabular}

Bank flux at least twice as small as our Nançay value (IC 4567, NGC 2998 and 5320); (2) the Nançay-Arecibo comparison shows one object with an Arecibo flux more than twice as large as the Nançay value (NGC 3442) and 5 objects with an Arecibo flux less than twice the Nançay value (in order of increasing Arecibo flux: NGC 3166, 3786, 3227, UGC 6806 and NGC 5533); (3) a comparison between our Nançay data and profiles obtained earlier with the same telescope shows 2 objects with a literature Nançay flux more than twice as large as our value (in order of increasing flux: NGC 3187 and 5383) and one with a literature Nançay flux less than twice our value: GC 3169.

\subsection{H I profile linewidths}

In Figs. 3 and 4 we compare, respectively, our directly measured $W_{50}$ and $W_{20}$ linewidths with values from other sources (see Tables 6 and 7). Panels $a, b$ and $c$ show our linewidths versus, respectively, previous Arecibo, Green Bank 90-m and Nançay measurements. Cases where we measured an $\mathrm{H}$ I linewidth more than twice as wide or more than twice as narrow as a published linewidth are discussed in Sect. 4.1; these discrepancies are all related to the presence of extended H I structures in the groups.

The cases in which large differences between measured linewidths were found are: (1.) The Nançay-Green Bank comparison shows 2 galaxies or close galaxy pairs with a Green Bank $W_{50}$ measurement more than twice as large as the Nançay value: NGC 3166 and $3226 / 7$ (in order of increasing linewidth); (2) the Nançay-Arecibo comparison shows one object with an Arecibo $W_{50}$ measurement more than twice as large as the Nançay value (NGC 3226/7) and one with a $W_{20}$ more than twice as large as the Nançay value: NGC 3166.

\section{Summary and conclusions}

The Nançay observations presented in this paper have provided $\mathrm{H}$ I line profiles and values or limits on gas masses and H I mass-to-light ratios for 91 members of loose groups. Five of the 11 galaxies in the sample which had not been observed previously, were detected by us (NGC 2852, NGC 5596, NGC 5934, UGC 6070 and UGC 6545) as well as 4 of the 10 galaxies which had not been detected previously (NGC 3193, NGC 5326, NGC 5380 and UGC 8736).

For galaxies which had been previously observed in the $\mathrm{H}$ I line, we generally find good agreement in radial velocities, while a larger scatter is seen for the $\mathrm{H}$ I line width parameters, especially $W_{20}$. The line widths are sensitive to the degree of resolution of the target galaxies, especially for the relatively small Arecibo beam, to the presence of tidal debris within the beams and to the quality of the data, in particular for $W_{20}$. The largest scatter is found in integrated H I fluxes, where differences of $~ 50 \%$ are common between various observations obtained with the Green Bank 90-m, Arecibo 305-m and Nançay telescopes. Our knowledge of the $\mathrm{H}$ I content of even the giant members of relatively nearby groups of galaxy is rather poor.

In a future paper we will analyze the $\mathrm{HI}$, optical and near-infrared properties of the "interacting" and "control" galaxy groups. We also plan to correlate H i properties with galaxy structural characteristics as determined from optical and near-infrared observations. 
Acknowledgements. We would like to thank the referee, Dr. M. S. Roberts, for his comments. The Unité Scientifique Nançay of the Observatoire de Paris is associated as Unité de Service et de Recherche (USR) No. B704 to the French Centre National de Recherche Scientifique (CNRS). Nançay also gratefully acknowledges the financial support of the Département du Cher, the European Community, the FNADT and the Région Centre. This research has made use of the Lyon-Meudon Extragalactic Database (LEDA) supplied by the LEDA team at the CRAL-Observatoire de Lyon (France), as well as of the NASA/IPAC Extragalactic Database (NED) which is operated by the Jet Propulsion Laboratory, California Institute of Technology, under contract with the National Aeronautics and Space Administration. Partial support for this program was provided through NASA grant NAG5-7040 and NSF grant AST 99-73812 to P.M.M. and through NSF grant AST-9875008 to E.M.W.

\section{References}

Allen, R. J., Goss, W. M., Sancisi, R., Sullivan, W. T., \& van Woerden, H. 1974, in The Formation and Dynamics of Galaxies, IAU Symp. 58, ed. J. R. Shakeshaft (Reidel: Dordrecht), 425

Bahcall, N. A., Harris, D. E., \& Rood, H. J. 1984, ApJ, 284, 29

Balkowski, C. 1979, A\&A, 78, 190

Balkowski, C., \& Chamaraux, P. 1983, A\&AS, 51, 331

Batuski, D. J., Hanisch, R. J., \& Burns, J. O. 1992, AJ, 103, 1077

Begeman, K. 1987, H I rotation curves of spiral galaxies, Ph.D. Thesis, University of Groningen

Bicay, M. D., \& Giovanelli, R. 1987, AJ, 93, 1326

Biermann, P., Clarke, J. N., \& Fricke, K. J. 1979, A\&A, 75, 7

Biermann, P., \& Kronberg, P. P. 1984, in Clusters and Groups of Galaxies, ed. F. Mardirossian, G. Giuricin, \& M. Mezzetti (Reidel: Dordrecht), 395

Bottinelli, L., Chamaraux, P., Gouguenheim, L., \& Lauqué, R. 1970, A\&A, 6, 453

Bottinelli, L., Gouguenheim, L., \& Paturel, G. 1980, A\&A, 88, 32

Bottinelli, L., Gouguenheim, L., \& Paturel, G. 1982, A\&A, 113, 61

Broeils, A. H. 1992, Dark and visible matter in spiral galaxies, Ph.D. Thesis, University of Groningen

Broeils, A. H., \& van Woerden, H. 1994, A\&AS, 107, 129

Chamaraux, P. 1977, A\&A, 60, 67

Chamaraux, P., Balkowski, C., \& Fontanelli, P. 1987, A\&AS, 69,263

Chamaraux, P., Balkowski, C., \& Gérard, E. 1980, A\&A, 83, 38

Chengalur, J. N., Salpeter, E. E., \& Terzian, Y. 1993, ApJ, 419,30

Chengalur, J. N., Salpeter, E. E., \& Terzian, Y. 1994, AJ, 107, 1984

Clemens, M. S., Baxter, K. M., Alexander, P., \& Green, D. A. 1999, MNRAS, 308, 364

Cole, G. H. J., Pedlar, A., Mundell, C. G., Gallimore, J. F., \& Holloway, A. J. 1998, MNRAS, 301, 782

Corsini, E. M., Pizzella, A., Funes, J. G., Vega Beltran, J. C., \& Bertola, F. 1998, A\&A, 337, 80

Davies, R. D. 1980, private communication
Davis, D. S., Mulchaey, J. S., \& Muchotzsky, R. F. 1999, ApJ, 511,34

Davis, L. E., \& Seaquist, E. R. 1983, ApJS, 53, 269

Dell'Antonio, I., Bothun, G. D., \& Geller, M. J. 1996, AJ, 112, 1759

de Vaucouleurs, G., de Vaucouleurs, A., Corwin, et al. 1991, Third Reference Catalogue of Bright Galaxies (New York: Springer) (RC3)

Dickel, J. R., \& Rood, H. J. 1978, ApJ, 223, 391

Duprie, K., \& Schneider, S. E. 1996, AJ, 112, 937

Eder, J., Giovanelli, R., \& Haynes, M. P. 1991, AJ, 102, 572

Falco, E. E., Kurtz, M. J., Geller, M. J., et al. 1999, PASP, 111,438

Fisher, J. R., \& Tully, R. B. 1981, ApJS, 47, 139

Ford Jr., W. K., Rubin, V. C., \& Roberts, M. S. 1971, AJ, 76, 22

Fouqué, P., Bottinelli, L., Durand, N., Gouguenheim, L., \& Paturel, G. 1990, A\&AS, 86, 473

Freudling, W. 1995, A\&AS, 112, 429

Fukazawa, Y., Makishima, K., Matsushita, K., et al. 1996, PASJ, 48, 395

Gallagher, J. S., Littleton, F. E., \& Matthews, L. D. 1995, AJ, 109, 2003

Garcia, A. M. 1993, A\&AS, 100, 47

Garcia, A. M., Bottinelli, L., Garnier, R., Gouguenheim, L., \& Paturel, G. 1994, A\&AS, 107, 265

Garwood, R. W., Helou, G., \& Dickey, J. M. 1987, ApJ, 322, 88

Gavazzi, G. 1987, ApJ, 320, 96

Geller, M. J., \& Huchra, J. P. 1983, ApJS, 52, 61

Giovanardi, C., Krumm, N., \& Salpeter, E. E. 1983, AJ, 88, 1719

Giovanardi, C., \& Salpeter, E. E. 1985, ApJS, 58, 623

Haynes, M. P. 1981, AJ, 86, 1126

Haynes, M. P., Giovanelli, R., \& Chincarini, G. L. 1985, ARA\&A, 22, 445

Haynes, M. P., Giovanelli, R., Starosta, B. M., \& Magri, C. 1988, AJ, 95, 607

Haynes, M. P., Jore, K. P., Barrett, E. A., Broeils, A. H., \& Murray, B. M. 2000, AJ, 120, 703

Heckman, T. M., Balick, B., \& Breugel, W. J. M. 1983, AJ, 88,583

Heckman, T. M., Balick, B., \& Sullivan III, W. T. 1978, ApJ, 224,745

Helou, G., Giovanardi, C., Salpeter, E. E., \& Krumm, N. 1981, ApJS, 46, 267

Helou, G., Salpeter, E. E., \& Terzian, Y. 1982, AJ, 87, 1443

Hernquist, L., Katz, N. S., \& Weinberg, D. H. 1995, ApJ, 442, 57

Hickson, P. 1982, ApJ, 255, 382

Hickson, P., Kindl, E., \& Weinberg, D. H. 1988, ApJ, 331, 64

Hoffman, G. L., Salpeter, E. E., Lamphier, C., \& Roos, T. 1992, ApJ, 388, L5

Huchra, J., Davis, M., Latham, D., \& Tonry, J. 1983, ApJS, 52,89

Huchra, J. P., Geller, M. J., \& Corwin, H. G. Jr. 1995, ApJS, 99, 391

Huchtmeier, W. K. 1982, A\&A, 110, 121

Huchtmeier, W. K. 1994, A\&A, 286, 389

Huchtmeier, W. K. 1997, A\&A, 319, 401

Huchtmeier, W. K., \& Bohnenstengel, H. D. 1975, A\&A, 44, 479 
Huchtmeier, W. K., \& Richter, O.-G. 1989, A General Catalogue of $\mathrm{HI}$ Observations of Galaxies (Springer, Heidelberg)

Huchtmeier, W. K., Sage, L. J., \& Henkel, C. 1995, A\&A, 300, 675

Jackson, J., Barett, A. H., Amstrong, J. T., \& Ho, P. T. P. 1987, AJ, 93, 531

Jore, K. P., Broeils, A. H., \& Haynes, M. P. 1996, AJ, 112, 438

Kamphuis, J., Sijbring, D., \& van Albada, T. S. 1996, A\&AS, 116,15

Karachentsev, I. D. 1980, ApJS, 44, 137

Knapp, G. R., Kerr, F. J., \& Henderson, A. P. 1979, ApJ, 234, 448

Knapp, G. R., Kerr, F. J., \& Williams, B. A. 1978, ApJ, 222, 800

Kraan-Korteweg, R. C., van Driel, W., Briggs, F., Binggeli, B., \& Mostefaoui, T. I. 1999, A\&AS, 135, 255

Krumm, N., \& Salpeter, E. E. 1980, AJ, 85, 1312

Lake, G., \& Schommer, R. A. 1984, ApJ, 280, 107

Lewis, B. M. 1985, ApJ, 292, 451

Lewis, B. M. 1987, ApJS, 63, 515

Lewis, B. M., \& Davies, R. D. 1973, MNRAS, 165, 213

Lewis, B. M., Helou, G., \& Salpeter, E. E. 1985, ApJS, 59, 161

Lo, K. Y., \& Sargent, W. L. 1979, ApJ, 227, 756

Magri, C. 1994, AJ, 108, 896

Mahdavi, A., Boehringer, H., Geller, M., \& Ramella, M. 1997, ApJ, 483, 68

Mamon, G. A. 1986, ApJ, 307, 426

Marcum, P. 1994, Star Formation Structure and Evolution of Galaxies in Loose Groups: the Environmental Connection, Ph.D. Thesis (University of Wisconsin, Madison)

Martin, M. C. 1998, A\&AS, 131, 73

Matthews, L. D., van Driel, W., \& Gallagher, J. S. 1998, AJ, 116,1196

Matthews, L. D., \& van Driel, W., 2000, A\&AS, 143, 421

Matthews, L. D., van Driel, W., \& Monnier-Ragaigne, D. 2001, A\&AS, 365, 1

Mihos, J. C., Walker, I. R., Hernquist, L., Mendes de Olivera, C., \& Bolte, M. 1995, ApJ, 447, L87

Mirabel, I. F. 1982, ApJ, 260, 75

Mirabel, I. F., \& Sanders, D. B. 1988, ApJ, 335, 104

Mirabel, I. F., \& Wilson, A. S. 1984, ApJ, 277, 92

Mould, J. R., Akeson, R. L., Bothun, G. D., et al. 1993, ApJ, 409, 14

Mulchaey, J. S., \& Zabludoff, A. J. 1998, ApJ, 496, 73

Mulchaey, J., Davis, D., Mushotzky, R., \& Burstein, D. 1996, ApJ, 456, 80

Mundell, C. G., Pedlar, A., Axon, D. J., Meaburn, J., \& Unger, S. W., 1995, MNRAS, 277, 641

Nolthenius, R. A. 1993, ApJS, 85, 1

Nordgren, T. E., Chengalur, J. N., Salpeter, E. E., \& Terzian, Y. 1997, AJ, 114, 913
Oosterloo, T., \& Shostak, S. 1993, A\&AS, 99, 379

Ostriker, J. P., Lubin, L. M., \& Hernquist, L. 1995, ApJ, 444, L61

Paturel, G. 1998, private communication

Peterson, C. J., Rubin, V. C., Ford Jr., W. K., \& Thonnard, N. 1978, ApJ, 219, 31

Peterson, S. D. 1979, ApJS, 40, 527

Peterson, S. D., \& Shostak, G. S. 1974, AJ, 79, 767

Rhee, M.-H., \& van Albada, T. S. 1996, A\&AS, 115, 407

Richter, O.-G., \& Huchtmeier, W. K. 1991, A\&AS, 87, 425

Richter, O.-G., \& Sancisi, R. 1994, A\&A, 290, L9

Rood, H. J, \& Dickel, J. R. 1976, ApJ, 205, 346

Rose, J. A. 1977, ApJ, 211, 311

Rose, J. A. 1979, ApJ, 231, 10

Sancisi, R., Allen, R. J., \& Sullivan III, W. T. 1979, A\&A, 78, 217

Sanduleak, N., \& Pesch, P. 1987, ApJS, 63, 809

Schneider, S. E., Helou, G., Salpeter, E. E., \& Terzian, Y. 1986a, AJ, 92, 742

Schneider, S. E., Salpeter, E. E., \& Terzian, Y. 1986b, AJ, 91, 13

Schneider, S. E., Thuan, T. X., Magri, C., \& Wadiak, J. E. 1990, ApJS, 72, 245

Scodeggio, M., \& Gavazzi, G. 1993, ApJ, 409, 110

Shostak, G. S. 1975, ApJ, 198, 527

Shostak, G. S. 1978, A\&A, 68, 321

Silverglate, P. R., \& Krumm, N. 1978, ApJ, 224, L98

Staveley-Smith, L., \& Davies, R. D. 1987, MNRAS, 224, 953

Staveley-Smith, L., \& Davies, R. D. 1988, MNRAS, 231, 833

Sulentic, J. W., \& Arp, H. 1983, AJ, 88, 489

Theureau, G., Bottinelli, L., Coudreau-Durand, N., et al. 1998, A\&AS, 130, 333

Thonnard, N., Rubin, V. C., Ford Jr., W. K., \& Roberts, M. S. 1978, AJ, 83, 1564

Thuan, T. X., \& Martin, G. E. 1981, ApJ, 247, 823

Tifft, W. G., \& Cocke, W. J. 1988, ApJS, 67, 1

van der Burg, G. 1985, A\&AS, 62, 147

van Driel, W., \& van Woerden, H. 1991, A\&A, 243, 71

van Moorsel, G. A. 1983, A\&AS, 53, 271

Wardle, M., \& Knapp, G. R. 1985, AJ, 91, 23

Wevers, B. M. H. R., van der Kruit, P. C., \& Allen, R. J. 1986, A\&AS, 66, 505

Williams, B. A. 1985, ApJS, 290, 462

Williams, B. A., \& Rood, H. J. 1987, ApJS, 63, 265

Williams, B. A., McMahon, P. M., \& van Gorkom, J. H. 1991, AJ, 101, 1957

Xue, Y.-J., \& Wu, X.-P. 2000, ApJ, 538, 65

Yun, M. S., Ho, P. T. P., \& Lo, K. Y. 1994, Nature, 372, 530

Zwaan, M. A. 2000, Atomic Hydrogen in the Local Universe, Ph.D. Thesis, University of Groningen

Zwaan, M. A. 2001, MNRAS, 325, 1142 